\title{
Shifted Darboux transformations of the generalized Jacobi matrices, I
}

\author{
IVAN M. KOVALYOV
}

(Presented by I. I. Skrypnik)

\begin{abstract}
Let $\mathfrak{J}$ be a monic generalized Jacobi matrix, i.e. a threediagonal block matrix of a special form. We find conditions for a monic generalized Jacobi matrix $\mathfrak{J}$ to admit a factorization $\mathfrak{J}=\mathfrak{L} \mathfrak{U}+\alpha I$ with $\mathfrak{L}$ and $\mathfrak{U}$ being lower and upper triangular two-diagonal block matrices of the special form. In this case the shifted Darboux transformation without parameter of $\mathfrak{J}$ defined by $\mathfrak{J}^{(p)}=\mathfrak{U} \mathfrak{L}+\alpha I$ is shown to be also a monic generalized Jacobi matrix. Analogues of Christoffel formulas for polynomials of the first and second kind, corresponding to the Darboux transformation $\mathfrak{J}^{(p)}$ are found.
\end{abstract}

2010 MSC. Primary 47B36; Secondary 47B50; 42C05; 15A23.

Key words and phrases. Darboux transformation, indefinite inner product, $m$ - function, monic generalized Jacobi matrix, orthogonal polynomials, triangular factorization.

\section{Introduction}

Let $\mathfrak{S}$ be a linear functional defined on the linear space

$$
\mathcal{P}=\operatorname{span}\left\{\lambda^{j}: j \in \mathbb{Z}_{+}:=\mathbb{N} \cup\{0\}\right\}
$$

by the equality

$$
\mathfrak{S}\left(\lambda^{j}\right)=\mathfrak{s}_{j}, \quad j \in \mathbb{Z}_{+} .
$$

The numbers $\mathfrak{s}_{j}$ are called the $j$-th moment associated with the linear functional $\mathfrak{S}$. The moment sequence $\mathfrak{s}=\left\{\mathfrak{s}_{j}\right\}_{j=0}^{\infty}$ has got a set of normal indices defined by

$$
\mathcal{N}(\mathfrak{s})=\left\{\mathfrak{n}_{j}: \mathbf{d}_{\mathfrak{n}_{j}} \neq 0, j=1,2, \ldots\right\}, \quad \mathbf{d}_{n_{j}}=\operatorname{det}\left(\mathfrak{s}_{i+k}\right)_{i, k=0}^{n_{j}-1} .
$$

Received 20.10.2018 
As follows from $(1.3) \mathfrak{n}_{j}$ is a normal index of $\mathfrak{s}$ if and only if

$$
\operatorname{det}\left(\begin{array}{ccc}
\mathfrak{s}_{0} & \cdots & \mathfrak{s}_{\mathfrak{n}_{j}-1} \\
\cdots & \cdots & \cdots \\
\mathfrak{s}_{\mathfrak{n}_{j}-1} & \cdots & \mathfrak{s}_{2 \mathfrak{n}_{j}-2}
\end{array}\right) \neq 0
$$

It is well known (see [7], [21]) that the sequence of monic polynomials $\left\{P_{j}(\lambda)\right\}_{j=0}^{\infty}$ is orthogonal polynomials with respect to the linear functional $\mathfrak{S}$. Furthermore (see $[5,7]$ ), the sequence $\left\{P_{j}(\lambda)\right\}_{j=0}^{\infty}$ is associated with the following monic generalized Jacobi matrix

$$
\mathfrak{J}=\left(\begin{array}{cccc}
\mathfrak{C}_{\mathfrak{p}_{0}} & \mathfrak{D}_{0} & & \\
\mathfrak{B}_{1} & \mathfrak{C}_{\mathfrak{p}_{1}} & \mathfrak{D}_{1} & \\
& \mathfrak{B}_{2} & \mathfrak{C}_{\mathfrak{p}_{2}} & \ddots \\
& & \ddots & \ddots
\end{array}\right)
$$

where the entries are the blocks of the special type.

As was known (see [15]), the matrix $\mathfrak{J}$ admits the $\mathfrak{L} \mathfrak{U}$ - factorization

$$
\mathfrak{J}=\mathfrak{L} \mathfrak{U},
$$

if and only if

$$
P_{\mathfrak{n}_{j}}(0) \neq 0,
$$

where $\mathfrak{L}$ and $\mathfrak{U}$ are lower and upper triangular block matrices. Multiplying the matrices $\mathfrak{L}$ and $\mathfrak{U}$ in reverse order, we obtain

$$
\mathfrak{J}^{(p)}=\mathfrak{U} \mathfrak{L}
$$

is the generalized Jacobi matrix associated with a linear functional

$$
\mathfrak{S}^{(p)}(p(\lambda))=\mathfrak{S}(\lambda p(\lambda)), \quad p(\lambda) \text { is a polynomial. }
$$

A sequence of the monic polynomials $\left\{P_{j}^{(p)}(\lambda)\right\}_{j=0}^{\infty}$ associated with the functional $\mathfrak{S}^{(p)}$ is called a Christoffel transform of $\left\{P_{j}(\lambda)\right\}_{j=0}^{\infty}$ (see [4,23]). In this case, the transformation (1.6), (1.8) is called a Darboux transformation (without parameter). Darboux transformation was also study for the Stieltjes strings (see [10]). The Darboux transformation was also studied for the Stieltjes strings (see [10]). The Darboux transformation of the discrete analogue of the Laguerre operator was investigated in [17].

It is worth noting, when $\mathfrak{C}_{\mathfrak{p}_{j}}$ are $1 \times 1$ matrices (i.e. $\mathfrak{J}$ is a monic Jacobi matrices), the Darboux transformation without parameter (1.6), 
(1.8) was studied in [2]. In the case, $\mathfrak{C}_{\mathfrak{p}_{j}}$ are $1 \times 1$ or $2 \times 2$ matrices, the Darboux transformation without parameter (1.6), (1.8) was studied in [8]. The results of $[2,8]$ are the particular case of [15].

As was shown in [6], the monic generalized Jacobi matrices $\mathfrak{J}$ admits a shifted Darboux transformation (without parameter), i.e.

$$
\mathfrak{J}=\mathfrak{L} \mathfrak{U}+\alpha I \rightarrow \mathfrak{U} \mathfrak{L}+\alpha I=\mathfrak{J}^{(p)} .
$$

if and only if

$$
P_{\mathfrak{n}_{j}}(\alpha) \neq 0
$$

and its diagonal blocks $\mathfrak{C}_{\mathfrak{p}_{j}}$ are $2 \times 2$ matrices. In this case, $\mathfrak{J}^{(p)}$ is the monic Jacobi matrices associated with the sequence of the monic polynomials $\left\{P_{j}^{(p)}(\lambda)\right\}_{j=0}^{\infty}$. The polynomials $P_{j}^{(p)}(\lambda)$ are orthogonal with respect to the linear functional

$$
\mathfrak{S}^{(p)}(\lambda)=(\lambda-\alpha) \mathfrak{S}(\lambda) .
$$

$\left\{P_{j}^{(p)}(\lambda)\right\}_{j=0}^{\infty}$ is a special case of the Christoffel transformation (see [24]).

The present paper is generalization of the papers $[2,6,8,15]$ and [16]. It is shown that every generalized Jacobi matrix $\mathfrak{J}$, which satisfies conditions similar to (1.11), admits an $\mathfrak{L} \mathfrak{U}$-factorization $\mathfrak{J}+\alpha I=\mathfrak{L} \mathfrak{U}$, with lowertriangular and upper-triangular two-diagonal block matrices $\mathfrak{L}$ and $\mathfrak{U}$. It turns out that the monic generalized Jacobi matrix $\mathfrak{J}^{(p)}$, associated with the linear functional $\mathfrak{S}^{(p)}$ defined by (1.12), can be represented as $\mathfrak{J}^{(p)}=\mathfrak{U} \mathfrak{L}+\alpha I$. This monic generalized Jacobi matrix $\mathfrak{J}^{(p)}$ is called the shifted Darboux transformation without parameter of $\mathfrak{J}$.

Darboux transformation of the orthogonal polynomials is actual. It is also studied of the matrix orthogonal polynomials associated with the some differential operator (see $[3,11]$ ).

In this paper we consider the firs part of shifted Darboux transformation of generalized Jacobi matrices, namely, the transformation without parameter is study. The shifted Darboux transformation will be applied to the indefinite Stieltjes moment problem. These results will be published elsewhere.

\section{Monic generalized Jacobi matrices associated with non-quasi-definite functional}

Let $\left\{\mathfrak{s}_{j}\right\}_{j=0}^{\infty}$ be a sequence of real moments and let $\mathfrak{S}$ be a linear functional defined on the linear space $\mathcal{P}=\operatorname{span}\left\{\lambda^{j}: j \in \mathbb{Z}_{+}\right\}$by (1.2). 
We denote the first nontrivial moment $\varepsilon_{0}:=\mathfrak{s}_{\mathfrak{n}_{1}-1}$, i.e. $\mathfrak{s}_{k}=0$ for all $k<\mathfrak{n}_{1}-1$. For example, if $\mathfrak{n}_{1}=1$, then $\mathfrak{s}_{0} \neq 0$ and $\varepsilon_{0}=\mathfrak{s}_{0}$.

Using moment sequence $\left\{\mathfrak{s}_{j}\right\}_{j=0}^{\infty}$, we can construct the polynomials of the first and the second kind (see $[1,5]$ ), defined by for all $j \in \mathbb{N}$

$$
P_{\mathfrak{n}_{j}}(\lambda)=\frac{1}{\mathbf{d}_{\mathfrak{n}_{j}}}\left|\begin{array}{cccc}
\mathfrak{s}_{0} & \mathfrak{s}_{1} & \cdots & \mathfrak{s}_{\mathfrak{n}_{j}} \\
\cdots & \cdots & \cdots & \cdots \\
\mathfrak{s}_{\mathfrak{n}_{j}-1} & \mathfrak{s}_{\mathfrak{n}_{j}} & \cdots & \mathfrak{s}_{2 \mathfrak{n}_{j}-1} \\
1 & \lambda & \cdots & \lambda^{\mathfrak{n}_{j}}
\end{array}\right|, Q_{\mathfrak{n}_{j}}(\lambda)=\mathfrak{S}_{t}\left(\frac{P_{\mathfrak{n}_{j}}(\lambda)-P_{\mathfrak{n}_{j}}(t)}{\lambda-t}\right)
$$

The polynomials $P_{\mathfrak{n}_{j}}(\lambda)$ and $Q_{\mathfrak{n}_{j}}(\lambda)$ are solutions of a system of difference equations (see $[7,21]$ )

$$
\mathfrak{b}_{j} y_{\mathfrak{n}_{j-1}}(\lambda)-\mathfrak{p}_{j}(\lambda) y_{\mathfrak{n}_{j}}(\lambda)+y_{\mathfrak{n}_{j+1}}(\lambda)=0 \quad\left(\mathfrak{b}_{0}=\varepsilon_{0}\right)
$$

subject to the initial conditions

$$
P_{\mathfrak{n}_{-1}}(\lambda) \equiv 0, P_{\mathfrak{n}_{0}}(\lambda) \equiv 1, Q_{\mathfrak{n}_{-1}}(\lambda) \equiv-1, Q_{\mathfrak{n}_{0}}(\lambda) \equiv 0,
$$

where $\mathfrak{b}_{j} \in \mathbb{R} \backslash\{0\}, \mathfrak{p}_{j}(\lambda)=\lambda^{\ell_{j}}+\mathfrak{p}_{\ell_{j}-1}^{(j)} \lambda^{\ell_{j}-1}+\ldots+\mathfrak{p}_{1}^{(j)} \lambda+\mathfrak{p}_{0}^{(j)}$ are monic polynomials of degree $\ell_{j}=\mathfrak{n}_{j+1}-\mathfrak{n}_{j}$ and generating polynomials of the following generalized Jacobi matrix $\mathfrak{J}, j \in \mathbb{Z}_{+}$.

One can associate with the system (2.2) the so-called monic generalized Jacobi matrix (GJM) (see $[7,8])$

$$
\mathfrak{J}=\left(\begin{array}{cccc}
\mathfrak{C}_{\mathfrak{p}_{0}} & \mathfrak{D}_{0} & & \\
\mathfrak{B}_{1} & \mathfrak{C}_{\mathfrak{p}_{1}} & \mathfrak{D}_{1} & \\
& \mathfrak{B}_{2} & \mathfrak{C}_{\mathfrak{p}_{2}} & \ddots \\
& & \ddots & \ddots
\end{array}\right)
$$

where the diagonal entries are companion matrices associated with the some real polynomials $\mathfrak{p}_{j}(\lambda)$ (see [18])

$$
\mathfrak{C}_{\mathfrak{p}_{j}}=\left(\begin{array}{ccccc}
0 & 1 & 0 & \cdots & 0 \\
0 & 0 & 1 & \ddots & \vdots \\
\vdots & \ddots & \ddots & \ddots & 0 \\
0 & \cdots & 0 & 0 & 1 \\
-\mathfrak{p}_{0}^{(j)} & -\mathfrak{p}_{1}^{(j)} & \cdots & -\mathfrak{p}_{\ell_{j}-2}^{(j)} & -\mathfrak{p}_{\ell_{j}-1}^{(j)}
\end{array}\right) \text { are } \ell_{j} \times \ell_{j} \text { matrices }
$$


$\mathfrak{D}_{j}$ and $\mathfrak{B}_{j+1}$ are $\ell_{j} \times \ell_{j+1}$ and $\ell_{j+1} \times \ell_{j}$ matrices, respectively, such that

$$
\mathfrak{D}_{j}=\left(\begin{array}{cccc}
0 & 0 & \cdots & 0 \\
\vdots & \vdots & \cdots & \vdots \\
0 & 0 & \cdots & 0 \\
1 & 0 & \cdots & 0
\end{array}\right), \mathfrak{B}_{j+1}=\left(\begin{array}{cccc}
0 & 0 & \cdots & 0 \\
\vdots & \vdots & \cdots & \vdots \\
0 & 0 & \cdots & 0 \\
\mathfrak{b}_{j+1} & 0 & \cdots & 0
\end{array}\right), \mathfrak{b}_{j+1} \neq 0, j \in \mathbb{Z}_{+} .
$$

The matrix $\mathfrak{J}$ defined by (2.4)-(2.6) is called a GJM associated with the linear functional $\mathfrak{S}$. Sometimes $\mathfrak{J}$ is called a GJM associated with the sequence $\left\{\mathfrak{s}_{j}\right\}_{j=0}^{\infty}$ or the system (2.2) to emphasize connection with polynomials $\mathfrak{p}_{j}(\lambda)$ and numbers $\mathfrak{b}_{j+1}, j \in \mathbb{Z}_{+}$.

The shortened $G J M \mathfrak{J}_{[i, j]}$ is defined by

$$
\mathfrak{J}_{[i, j]}=\left(\begin{array}{cccc}
\mathfrak{C}_{\mathfrak{p}_{i}} & \mathfrak{D}_{i} & & \\
\mathfrak{B}_{i+1} & \mathfrak{C}_{i+1} & \ddots & \\
& \ddots & \ddots & \mathfrak{D}_{j-1} \\
& & \mathfrak{B}_{j} & \mathfrak{C}_{\mathfrak{p}_{j}}^{-1}
\end{array}\right), i \leq j \text { and } i, j \in \mathbb{Z}_{+}
$$

The following connection between the polynomials of the first and the second kind and the shortened GJM's can be found in [7]

$$
P_{\mathfrak{n}_{j}}(\lambda)=\operatorname{det}\left(\lambda-\mathfrak{J}_{[0, j-1]}\right) \quad \text { and } \quad Q_{\mathfrak{n}_{j}}(\lambda)=b_{0} \operatorname{det}\left(\lambda-\mathfrak{J}_{[1, j-1]}\right) .
$$

Let us define an inner product in the space $\ell_{\left[0, \mathfrak{n}_{j}-1\right]}^{2}$ by

$$
[x, y]=(G x, y)_{\ell_{\left[0, \mathfrak{n}_{j}-1\right]}^{2}},
$$

where $x, y \in \ell_{\left[0, \mathfrak{n}_{j}-1\right]}^{2}, G_{[0, j-1]}=\operatorname{diag}\left(b_{0} G_{0}, b_{0} b_{1} G_{1}, \ldots, b_{0} \ldots b_{j-1} G_{j-1}\right)$ and

$$
G_{i}=\left(\begin{array}{cccc}
\mathfrak{p}_{1}^{(i)} & \cdots & \mathfrak{p}_{\ell_{i}-1}^{(i)} & 1 \\
\vdots & \ddots & \ddots & \\
\mathfrak{p}_{\ell_{i}-1}^{(i)} & \ddots & & \\
1 & & & 0
\end{array}\right)^{-1} i=\overline{0, j-1}
$$

Let us set

$$
\begin{aligned}
& \mathbf{P}(\lambda)=\left(P_{0}(\lambda), P_{1}(\lambda), \ldots, P_{\mathfrak{n}_{j}}(\lambda), \ldots\right)^{T} \\
& \mathbf{Q}(\lambda)=\left(Q_{0}(\lambda), Q_{1}(\lambda), \ldots, Q_{\mathfrak{n}_{j}}(\lambda), \ldots\right)^{T},
\end{aligned}
$$


where $P_{\mathfrak{n}_{j}+k}(\lambda)=\lambda^{k} P_{\mathfrak{n}_{j}}(\lambda)$ and $Q_{\mathfrak{n}_{j}+k}(\lambda)=\lambda^{k} Q_{\mathfrak{n}_{j}}(\lambda)$, where $0 \leq k<$ $\mathfrak{n}_{j+1}-\mathfrak{n}_{j}$. Then it follows from (2.2)-(2.6), that

$$
(\mathfrak{J}-\lambda I) \mathbf{P}(\lambda)=0 \quad \text { and } \quad(\mathfrak{J}-\lambda I) \mathbf{Q}(\lambda)=(\underbrace{0, \ldots, 0, b_{0}}_{\ell_{0}}, 0, \ldots)^{T} .
$$

Definition 2.1. Let us define the $m$-function of the matrix $\mathfrak{J}$ by equality

$$
m_{[0, j-1]}(\lambda)=\left[\left(\mathfrak{J}_{[0, j-1]}^{T}-\lambda\right)^{-1} e_{0}, e_{0}\right],
$$

where $e_{0}=\left(\begin{array}{llll}1 & 0 & \cdots & 0\end{array}\right)^{T}$ is $\mathfrak{n}_{j} \times 1$ vector.

As was shown in [8, Proposition 6.1], [20, see formula (139)], [13]

$$
m_{[0, j-1]}(\lambda)=-\varepsilon_{0} \frac{\operatorname{det}\left(\lambda-\mathfrak{J}_{[1, j-1]}\right)}{\operatorname{det}\left(\lambda-\mathfrak{J}_{[0, j-1]}\right)}=-\frac{Q_{\mathfrak{n}_{j}}(\lambda)}{P_{\mathfrak{n}_{j}}(\lambda)}
$$

and $m_{[0, j-1]}(\lambda)$ admits the following asymptotic expansion

$$
m_{[0, j-1]}(\lambda)=-\frac{\mathfrak{s}_{0}}{\lambda}-\frac{\mathfrak{s}_{1}}{\lambda^{2}}-\cdots-\frac{\mathfrak{s}_{2 \mathfrak{n}_{j}-2}}{\lambda^{2 \mathfrak{n}_{j}-1}}+o\left(\frac{1}{\lambda^{2 \mathfrak{n}_{j}-1}}\right),
$$

where the moments $\mathfrak{s}_{k}$ are calculated by

$$
\mathfrak{s}_{k}=\left[\left(\mathfrak{J}_{[0, j-1]}^{T}\right)^{k} e_{0}, e_{0}\right], \quad k \leq 2 \mathfrak{n}_{j}-2 .
$$

\section{Shifted Darboux transformation without parameter}

In this section we study the shifted Darboux transformation without parameter of the monic generalized Jacobi matrices, which may apply even in the case, when some $P_{\mathfrak{n}_{j}}(0)=0$, that is not true of the Darboux transformation (without parameter) in [15]. The paper [15] is the particular case of this section ([15] has got the shift equals to zero). The realization of the shifted Darboux transformation without parameter is carried out using the following block lower and upper triangular matrices

$$
\mathfrak{L}=\left(\begin{array}{cccc}
\mathfrak{A}_{0} & 0 & & \\
\mathfrak{L}_{1} & \mathfrak{A}_{1} & 0 & \\
& \mathfrak{L}_{2} & \mathfrak{A}_{2} & \ddots \\
& & \ddots & \ddots
\end{array}\right) \quad \text { and } \quad \mathfrak{U}=\left(\begin{array}{cccc}
\mathfrak{U}_{0} & \mathfrak{D}_{0} & & \\
0 & \mathfrak{U}_{1} & \mathfrak{D}_{1} & \\
& 0 & \mathfrak{U}_{2} & \ddots \\
& & \ddots & \ddots
\end{array}\right)
$$


the diagonal blocks $\mathfrak{A}_{j}$ and $\mathfrak{U}_{j}$ are $\ell_{j} \times \ell_{j}$ matrices $\left(\mathfrak{u}_{j} \neq 0\right)$

$$
\mathfrak{A}_{j}=\left(\begin{array}{ccccc}
1 & 0 & 0 & \cdots & 0 \\
0 & 1 & 0 & \cdots & 0 \\
\vdots & \ddots & \ddots & \ddots & \vdots \\
0 & 0 & \cdots & 1 & 0 \\
\mathfrak{a}_{1}^{(j)} & \mathfrak{a}_{2}^{(j)} & \cdots & \mathfrak{a}_{\ell_{j}-1}^{(j)}-\alpha & 1
\end{array}\right), \mathfrak{U}_{j}=\left(\begin{array}{ccccc}
-\alpha & 1 & 0 & \cdots & 0 \\
0 & -\alpha & 1 & \ddots & \vdots \\
\vdots & \ddots & \ddots & \ddots & 0 \\
0 & \cdots & 0 & -\alpha & 1 \\
-\mathfrak{u}_{j} & 0 & \cdots & 0 & 0
\end{array}\right),
$$

the blocks $\mathfrak{L}_{j+1}$ and $\mathfrak{D}_{j}$ are $\ell_{j+1} \times \ell_{j}$ and $\ell_{j} \times \ell_{j+1}$ matrices, respectively

$$
\mathfrak{L}_{j+1}=\left(\begin{array}{cccc}
0 & 0 & \cdots & 0 \\
\vdots & \vdots & \cdots & \vdots \\
0 & 0 & \cdots & 0 \\
0 & 0 & \cdots & \mathfrak{l}_{j+1}
\end{array}\right), \mathfrak{l}_{j+1} \neq 0, \mathfrak{D}_{j}=\left(\begin{array}{cccc}
0 & 0 & \cdots & 0 \\
\vdots & \vdots & \cdots & \vdots \\
0 & 0 & \cdots & 0 \\
1 & 0 & \cdots & 0
\end{array}\right), j \in \mathbb{Z}_{+} .
$$

However, if $\ell_{j}=1$, then we mean

$$
\mathfrak{A}_{j}=(1) \quad \text { and } \quad \mathfrak{U}_{j}=\left(-\mathfrak{u}_{j}\right)
$$

if $\ell_{j}=\ell_{j+1}=1$, then

$$
\mathfrak{L}_{j+1}=\left(\mathfrak{l}_{j+1}\right) \quad \text { and } \quad \mathfrak{D}_{j}=(1) .
$$

Let us say that the matrix $\mathfrak{J}-\alpha I$ admits an $\mathfrak{L} \mathfrak{U}-$ factorization if $\mathfrak{J}-\alpha I$ is represented in the form $\mathfrak{J}-\alpha I=\mathfrak{L} \mathfrak{U}$, where $\mathfrak{L}$ and $\mathfrak{U}$ are given by (3.1)-(3.3) and $\alpha \in \mathbb{R}$.

Definition 3.1. Let $\mathfrak{J}$ be the monic generalized Jacobi matrix and let $\mathfrak{J}-\alpha I$ admit the $\mathfrak{L} \mathfrak{U}$-factorization of the form (3.1)-(3.3). Then the transformation

$$
\mathfrak{J}=\mathfrak{L} \mathfrak{U}+\alpha I \rightarrow \mathfrak{U} \mathfrak{L}+\alpha I \rightarrow=\mathfrak{J}^{(p)}
$$

is called a shifted Darboux transformation without parameter of $\mathfrak{J}$. The matrix $\mathfrak{J}^{(p)}$ is a monic generalized Jacobi matrix.

Lemma 3.1. Let $\mathfrak{J}$ be the monic generalized Jacobi matrix associated with the functional $\mathfrak{S}$ and let $\ell_{j}:=\mathfrak{n}_{j+1}-\mathfrak{n}_{j} \geq 1, j \in \mathbb{Z}_{+}$, where $\mathfrak{n}_{0}=0$ and $\left\{\mathfrak{n}_{j}\right\}_{j=1}^{\infty}$ is the set of normal indices of the sequence $\mathfrak{s}=\left\{\mathfrak{s}_{j}\right\}_{j=0}^{\infty}$. Let $\mathfrak{L}$ and $\mathfrak{U}$ be defined by (3.1)-(3.3) and $\alpha \in \mathbb{R}$. Then the matrix $\mathfrak{J}-\alpha I$ admits the $\mathfrak{L} \mathfrak{U}-$ factorization if and only if the system of equations

$$
-\mathfrak{l}_{j+1} \mathfrak{u}_{j}=\mathfrak{b}_{j+1},
$$




$$
\begin{gathered}
\mathfrak{l}_{j}-\mathfrak{u}_{j}=-\alpha-\mathfrak{p}_{0}^{(j)}, \quad \mathfrak{l}_{0}=0, \quad \ell_{j}=1, \\
\mathfrak{a}_{1}^{(j)}=-\mathfrak{p}_{1}^{(j)}, \quad-a\left(\mathfrak{a}_{1}^{(j)}-a\right)-\mathfrak{u}_{j}+\mathfrak{l}_{j}=-\mathfrak{p}_{0}^{(j)}, \quad \mathfrak{l}_{0}=0, \quad \ell_{j}=2, \quad(3.9) \\
\mathfrak{a}_{\ell_{j-1}}^{(j)}=-\mathfrak{p}_{\ell_{j-1}}^{(j)}, \mathfrak{a}_{\ell_{j-2}}^{(j)}-a\left(\mathfrak{a}_{\ell_{j-1}}^{(j)}-a\right)=-\mathfrak{p}_{\ell_{j-2}}^{(j)}, \mathfrak{a}_{\ell_{j-3}}^{(j)}-a \mathfrak{a}_{\ell_{j-2}}^{(j)}=-\mathfrak{p}_{\ell_{j-3}}^{(j)}, \ldots, \\
\mathfrak{a}_{1}^{(j)}-a \mathfrak{a}_{2}^{(j)}=-\mathfrak{p}_{1}^{(j)},-a \mathfrak{a}_{1}^{(j)}-\mathfrak{u}_{j}+\mathfrak{l}_{j}=-\mathfrak{p}_{0}^{(j)}, \quad \mathfrak{l}_{0}=0, \quad \ell_{j}>2
\end{gathered}
$$

is solvable for all $j \in \mathbb{Z}_{+}$.

Proof. Consider the product $\mathfrak{L} \mathfrak{U}$

$$
\mathfrak{L} \mathfrak{U}=\left(\begin{array}{cccc}
\mathfrak{A}_{0} \mathfrak{U}_{0} & \mathfrak{A}_{0} \mathfrak{D}_{0} & & \\
\mathfrak{L}_{1} \mathfrak{U}_{0} & \mathfrak{L}_{1} \mathfrak{D}_{0}+\mathfrak{A}_{1} \mathfrak{U}_{1} & \mathfrak{A}_{1} \mathfrak{D}_{1} & \\
& \mathfrak{L}_{2} \mathfrak{U}_{1} & \mathfrak{L}_{2} \mathfrak{D}_{1}+\mathfrak{A}_{2} \mathfrak{U}_{2} & \ddots \\
& & \ddots & \ddots
\end{array}\right),
$$

where $\mathfrak{L}_{j+1} \mathfrak{U}_{j}, \mathfrak{L}_{j+1} \mathfrak{D}_{j}$ are $\ell_{j+1} \times \ell_{j}$ and $\ell_{j+1} \times \ell_{j+1}$ matrices, respectively

$$
\mathfrak{L}_{j+1} \mathfrak{U}_{j}=\left(\begin{array}{cccc}
0 & 0 & \cdots & 0 \\
\vdots & \vdots & \ddots & \vdots \\
0 & 0 & \cdots & 0 \\
-\mathfrak{l}_{j+1} \mathfrak{u}_{j} & 0 & \cdots & 0
\end{array}\right), \mathfrak{L}_{j+1} \mathfrak{D}_{j}=\left(\begin{array}{cccc}
0 & 0 & \cdots & 0 \\
\vdots & \vdots & \ddots & \vdots \\
0 & 0 & \cdots & 0 \\
\mathfrak{l}_{j+1} & 0 & \cdots & 0
\end{array}\right)
$$

Comparing $\mathfrak{L}_{j+1} \mathfrak{U}_{j}$ with the block $\mathfrak{B}_{j+1}$ of the matrix $\mathfrak{J}$, we obtain

$$
-\mathfrak{l}_{j+1} \mathfrak{u}_{j}=\mathfrak{b}_{j+1} \quad \text { for all } j \in \mathbb{Z}_{+},
$$

i.e. (3.7) holds. The blocks $\mathfrak{A}_{j} \mathfrak{D}_{j}$ are $\ell_{j} \times \ell_{j+1}$ matrices and

$$
\mathfrak{A}_{j} \mathfrak{D}_{j}=\mathfrak{D}_{j} \quad j \in \mathbb{Z}_{+}
$$

(i) In this case we consider $\ell_{j}=1$, then

$$
\mathfrak{A}_{j} \mathfrak{U}_{j}=\left(-\mathfrak{u}_{j}\right)
$$

Therefore, if $j=0$, then we compare $\mathfrak{A}_{0} \mathfrak{U}_{0}$ with $\mathfrak{C}_{\mathfrak{p}_{0}}-(\alpha)$, we get

$$
\mathfrak{u}_{0}=\alpha+\mathfrak{p}_{0}^{(0)} \text {. }
$$

If $j>0$, then we compare $\mathfrak{L}_{j} \mathfrak{D}_{j-1}+\mathfrak{A}_{j} \mathfrak{U}_{j}$ with the block $\mathfrak{C}_{\mathfrak{p}_{j}}-(\alpha)$ and we obtain

$$
\mathfrak{l}_{j}-\mathfrak{u}_{j}=-\alpha-\mathfrak{p}_{0}^{(j)} .
$$


From (3.15) and (3.16), we have (3.8).

(ii) In this case we consider $\ell_{j}=2$, then

$$
\mathfrak{A}_{j} \mathfrak{U}_{j}=\left(\begin{array}{cc}
-\alpha & 1 \\
-\alpha\left(\mathfrak{a}_{1}^{(j)}-\alpha\right)-\mathfrak{u}_{j} & \mathfrak{a}_{1}^{(j)}-\alpha
\end{array}\right)
$$

If $j=0$, then we compare the block $\mathfrak{A}_{0} \mathfrak{U}_{0}$ with $\mathfrak{C}_{\mathfrak{p}_{0}}-\alpha I_{\ell_{0}}$, where $I_{\ell_{0}}$ is $\ell_{0} \times \ell_{0}$ identity matrix and we obtain

$$
\mathfrak{a}_{1}^{(0)}=-\mathfrak{p}_{1}^{(0)}, \quad-\alpha\left(\mathfrak{a}_{1}^{(0)}-\alpha\right)-\mathfrak{u}_{j}=-\mathfrak{p}_{0}^{(0)} .
$$

If $j>0$, then we compare the block $\mathfrak{L}_{j} \mathfrak{D}_{j-1}+\mathfrak{A}_{j} \mathfrak{U}_{j}$ with the block $\mathfrak{C}_{\mathfrak{p}_{j}}-\alpha I_{\ell_{j}}$ and we get

$$
\mathfrak{a}_{1}^{(j)}=-\mathfrak{p}_{1}^{(j)},-\alpha\left(\mathfrak{a}_{1}^{(j)}-\alpha\right)-\mathfrak{u}_{j}+\mathfrak{l}_{j}=-\mathfrak{p}_{0}^{(j)}
$$

Due to (3.18) and (3.19), the formula (3.9) holds.

(iii) In this case we consider $\ell_{j}>2$, then $\mathfrak{A}_{j} \mathfrak{U}_{j}=$

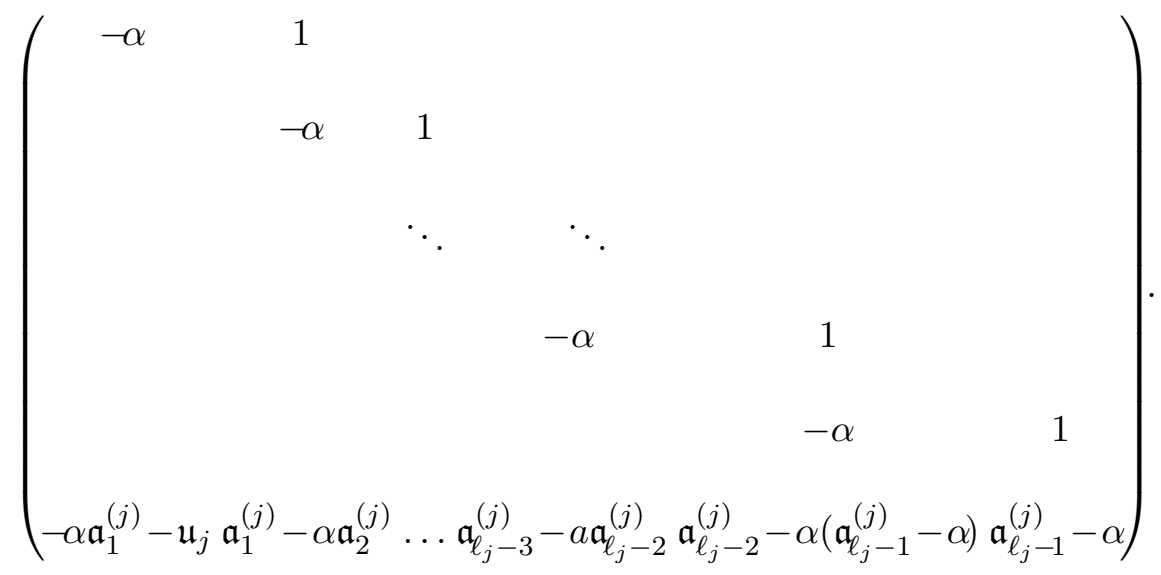

If $j=0$, then we compare the block $\mathfrak{A}_{0} \mathfrak{U}_{0}$ with the block $\mathfrak{C}_{\mathfrak{p}_{0}}-\alpha I_{\ell_{0}}$ and we obtain

$$
\begin{aligned}
& \mathfrak{a}_{\ell_{j-1}}^{(0)}=-\mathfrak{p}_{\ell_{j-1}}^{(0)}, \mathfrak{a}_{\ell_{j-2}}^{(0)}-\alpha\left(\mathfrak{a}_{\ell_{j-1}}^{(0)}-\alpha\right)=-\mathfrak{p}_{\ell_{j-2}}^{(0)}, \mathfrak{a}_{\ell_{j-3}}^{(0)}-\alpha \mathfrak{a}_{\ell_{j-2}}^{(0)}=-\mathfrak{p}_{\ell_{j-3}}^{(0)}, \ldots, \\
& \mathfrak{a}_{1}^{(0)}-\alpha \mathfrak{a}_{2}^{(0)}=-\mathfrak{p}_{1}^{(0)},-\alpha \mathfrak{a}_{1}^{(0)}-\mathfrak{u}_{j}=-\mathfrak{p}_{0}^{(0)}
\end{aligned}
$$


If $j>0$, then we compare the block $\mathfrak{L}_{j} \mathfrak{D}_{j-1}+\mathfrak{A}_{j} \mathfrak{U}_{j}$ with the $\mathfrak{C}_{\mathfrak{p}_{j}}-\alpha I_{\ell_{j}}$ and we obtain

$$
\begin{aligned}
& \mathfrak{a}_{\ell_{j-1}}^{(j)}=-\mathfrak{p}_{\ell_{j-1}}^{(j)}, \mathfrak{a}_{\ell_{j-2}}^{(j)}-\alpha\left(\mathfrak{a}_{\ell_{j-1}}^{(j)}-\alpha\right)=-\mathfrak{p}_{\ell_{j-2}}^{(j)}, \mathfrak{a}_{\ell_{j-3}}^{(j)}-\alpha \mathfrak{a}_{\ell_{j-2}}^{(j)}=-\mathfrak{p}_{\ell_{j-3}}^{(j)}, \ldots, \\
& \mathfrak{a}_{1}^{(j)}-\alpha \mathfrak{a}_{2}^{(j)}=-\mathfrak{p}_{1}^{(j)}, \quad-\alpha \mathfrak{a}_{1}^{(j)}-\mathfrak{u}_{j}+\mathfrak{l}_{j}=-\mathfrak{p}_{0}^{(j)} .
\end{aligned}
$$

Combining (3.20) and (3.21), we get (3.10).

Thus, we shown that if $\mathfrak{J}-\alpha I$ admits the $\mathfrak{L} \mathfrak{U}$-factorization of the form (3.1)-(3.3) then the system (3.7)-(3.10) is solvable. Conversely, if the system (3.7)-(3.10) is solvable, then $\mathfrak{J}-\alpha I=\mathfrak{L} \mathfrak{U}$. This completes the proof.

Lemma 3.2. Let $\mathfrak{J}$ be the GJM associated with the functional $\mathfrak{S}$ and let $\mathfrak{J}-\alpha I=\mathfrak{L} \mathfrak{U}$ be its $\mathfrak{L} \mathfrak{U}-$ factorization of the form (3.1))-(3.3) and let $P_{\mathfrak{n}_{j+1}}(\lambda)$ be polynomials of the first kind associated with $\mathfrak{J}$. Then

$$
P_{\mathfrak{n}_{j+1}}(\alpha)=\prod_{k=0}^{j} \mathfrak{u}_{k}, \text { for all } j \in \mathbb{Z}_{+} .
$$

Proof. Calculating $P_{\mathfrak{n}_{j+1}}(\alpha)=\operatorname{det}\left(\alpha I_{\mathfrak{n}_{j+1}}-\mathfrak{J}_{[0, j]}\right)$, we get

$$
P_{\mathfrak{n}_{j+1}}(a)=\left|\begin{array}{cccc}
\alpha I_{\ell_{0}}-\mathfrak{C}_{\mathfrak{p}_{0}} & -\mathfrak{D}_{0} & & \\
-\mathfrak{B}_{1} & \alpha I_{\ell_{1}}-\mathfrak{C}_{\mathfrak{p}_{1}} & \ddots & \\
& \ddots & \ddots & -\mathfrak{D}_{j-1} \\
& & -\mathfrak{B}_{j} & \alpha I_{\ell_{j}}-\mathfrak{C}_{\mathfrak{p}_{j}}
\end{array}\right|,
$$

we transform this determinant in the following way

(i) if $\ell_{k}>2$ and $k \leq j$, then we multiply the $\left(\mathfrak{n}_{k}+1\right)$-th row by $-\mathfrak{a}_{1}^{(k)}$ and add to the $\left(\mathfrak{n}_{k+1}\right)$-th row, the next we multiply the $\left(\mathfrak{n}_{k}+2\right)$ th row by $-\mathfrak{a}_{2}^{(k)}$ and add to the $\left(\mathfrak{n}_{k+1}\right)$-th row,..., but we multiply the $\left(\mathfrak{n}_{k+1}-1\right)$-th row by $-\left(\mathfrak{a}_{\ell_{k}-1}^{(k)}-\alpha\right)$ and add to the $\left(\mathfrak{n}_{k+1}\right)$-th row;

(ii) if $\ell_{k}=2$ and $k \leq j$, then we multiply the $\left(\mathfrak{n}_{k+1}-1\right)$-th row by $-\left(\mathfrak{a}_{1}^{(k)}-\alpha\right)$ and add to the $\left(\mathfrak{n}_{k+1}\right)$-th row;

(iii) if $\ell_{k}=1$ and $k \leq j$, then we don't transform $\left(\mathfrak{n}_{k+1}\right)$-th row.

Using (i), (ii) and (iii), we obtain

$$
P_{\mathfrak{n}_{j+1}}(a)=\left|\begin{array}{cccc}
\widetilde{\mathfrak{C}}_{\mathfrak{p}_{0}} & -\mathfrak{D}_{0} & & \\
-\mathfrak{B}_{1} & \widetilde{\mathfrak{C}}_{\mathfrak{p}_{1}} & \ddots & \\
& \ddots & \ddots & -\mathfrak{D}_{j-1} \\
& & -\mathfrak{B}_{j} & \widetilde{\mathfrak{C}}_{\mathfrak{p}_{j}}
\end{array}\right|
$$


where the blocks $\widetilde{\mathfrak{C}}_{\mathfrak{p}_{k}}$ are defined by

$$
\widetilde{\mathfrak{C}}_{\mathfrak{p}_{k}}=\left\{\begin{array}{c}
\left(\begin{array}{cccc}
a & -1 & \\
& \ddots & \ddots & \\
& & a & -1 \\
\mathfrak{u}_{k}-\mathfrak{l}_{k} & 0 & \cdots & 0 \\
\left(\mathfrak{u}_{k}-\mathfrak{l}_{k}\right) & , \quad \ell_{k}=1,
\end{array}, \quad \ell_{k} \geq 2 ; \quad k=\overline{0, j} \text { and } \mathfrak{l}_{0}=0 .\right.
\end{array}\right.
$$

Expanding (3.24) along the columns, which have only one element equals to -1 and others equal to zero and by [15, Lemma 3.3], we have

$$
P_{\mathfrak{u}_{j+1}}(a)=\left|\begin{array}{cccc}
\mathfrak{u}_{0} & -1 & & \\
\mathfrak{u}_{0} \mathfrak{l}_{1} & \mathfrak{u}_{1}-\mathfrak{l}_{1} & \ddots & \\
& \ddots & \ddots & -1 \\
& & \mathfrak{u}_{j-1} \mathfrak{l}_{j} & \mathfrak{u}_{j}-\mathfrak{l}_{j}
\end{array}\right|=\prod_{k=0}^{j} \mathfrak{u}_{k} .
$$

This completes the proof.

Corollary 3.1. Let $\mathfrak{J}$ be the GJM associated with the functional $\mathfrak{S}$ and let $\mathfrak{J}-\alpha I=\mathfrak{L} \mathfrak{U}$ be its $\mathfrak{L U}$-factorization of the form (3.1)-(3.3) and let $P_{\mathfrak{n}_{j+1}}(\lambda)$ be polynomials of the first kind associated with $\mathfrak{J}$. Then

$$
P_{\mathfrak{n}_{j+1}}(\alpha)=\mathfrak{u}_{j} \mathfrak{u}_{j-1} \ldots \mathfrak{u}_{j-k} P_{\mathfrak{n}_{j-k}}(\alpha), k \leq j \text { and } j, k \in \mathbb{Z}_{+} .
$$

Theorem 3.1. Let $\mathfrak{J}$ be the monic generalized Jacobi matrix associated with the functional $\mathfrak{S}$ and let $\ell_{j}:=\mathfrak{n}_{j+1}-\mathfrak{n}_{j} \geq 1, j \in \mathbb{Z}_{+}$, where $\mathfrak{n}_{0}=0$ and $\left\{\mathfrak{n}_{j}\right\}_{j=1}^{\infty}$ is the set of normal indices of the sequence $\mathfrak{s}=\left\{\mathfrak{s}_{j}\right\}_{j=0}^{\infty}$ and let $P_{\mathfrak{n}_{j}}(\lambda)$ be polynomials of the first kind associated with the sequence $\mathfrak{s}=\left\{\mathfrak{s}_{j}\right\}_{j=0}^{\infty}$ and $\alpha \in \mathbb{R}$. Then $\mathfrak{J}-a I$ admits the $\mathfrak{L U}-$ factorization of the form (3.1)-(3.3) if and only if

$$
P_{\mathfrak{n}_{j}}(\alpha) \neq 0 \quad \text { for all } j \in \mathbb{Z}_{+} .
$$

Furthermore

$\mathfrak{l}_{j+1}=-\frac{\mathfrak{b}_{j+1}}{\mathfrak{u}_{j}}, \mathfrak{u}_{j}=\frac{P_{\mathfrak{n}_{j+1}}(\alpha)}{P_{\mathfrak{n}_{j}}(\alpha)}, \mathfrak{u}_{0}=\left\{\begin{array}{r}\mathfrak{p}_{0}^{(0)}-a, \ell_{0}=1 ; \\ \mathfrak{p}_{0}^{(0)}-a\left(\mathfrak{a}_{1}^{(0)}-a\right), \ell_{0}=2 ; \\ \mathfrak{p}_{0}^{(0)}-a \mathfrak{a}_{1}^{(0)}, \ell_{0}>2 .\end{array}\right.$

Proof. Let $P_{\mathfrak{n}_{j}}(\alpha) \neq 0$ for all $j \in \mathbb{Z}_{+}$, then by Lemma 3.2 the system (3.29) is equivalent to the system (3.7)-(3.10). Consequently, by Lemma 3.1 the matrix $\mathfrak{J}-\alpha I$ admits the $\mathfrak{L} \mathfrak{U}$-factorization of the form (3.1)-(3.3). Conversely, let $\mathfrak{J}-\alpha I$ admit the $\mathfrak{L} \mathfrak{U}$-factorization of the form (3.1)-(3.3), then $P_{\mathfrak{n}_{j}}(\alpha) \neq 0$ for all $j \in \mathbb{Z}_{+}$(see Lemma 3.2). This completes the proof. 
Theorem 3.2. Let $\mathfrak{J}$ be the monic generalized Jacobi matrix associated with the functional $\mathfrak{S}$ and let $\mathfrak{J}-\alpha I=\mathfrak{L} \mathfrak{U}$ be its $\mathfrak{L} \mathfrak{U}$ - factorization of the form (3.1)-(3.3). Then the matrix $\mathfrak{J}^{(p)}=\mathfrak{U} \mathfrak{L}+\alpha I$ is the monic generalized Jacobi matrix.

Proof. Consider the product $\mathfrak{U} \mathfrak{L}$ of the matrices $\mathfrak{U}$ and $\mathfrak{L}$

$$
\mathfrak{U} \mathfrak{L}=\left(\begin{array}{cccc}
\mathfrak{U}_{0} \mathfrak{A}_{0}+\mathfrak{D}_{0} \mathfrak{L}_{1} & \mathfrak{D}_{0} \mathfrak{A}_{1} & & \\
\mathfrak{U}_{1} \mathfrak{L}_{1} & \mathfrak{U}_{1} \mathfrak{A}_{1}+\mathfrak{D}_{1} \mathfrak{L}_{2} & \mathfrak{D}_{1} \mathfrak{A}_{2} & \\
& \mathfrak{U}_{2} \mathfrak{L}_{2} & \mathfrak{U}_{2} \mathfrak{A}_{2}+\mathfrak{D}_{2} \mathfrak{L}_{3} & \ddots \\
& & \ddots & \ddots
\end{array}\right) .
$$

(i) Let $\ell_{j} \geq 2$ for all $j \in \mathbb{Z}_{+}$, then

$$
\mathfrak{U}_{j+1} \mathfrak{L}_{j+1}=\left(\begin{array}{cccc}
0 & \cdots & 0 & 0 \\
\vdots & \ddots & \vdots & \vdots \\
0 & \cdots & 0 & 0 \\
0 & \cdots & 0 & \mathfrak{l}_{j+1} \\
0 & \cdots & 0 & 0
\end{array}\right) \text { and } \mathfrak{D}_{j} \mathfrak{L}_{j+1}=\left(\begin{array}{ccc}
0 & \cdots & 0 \\
\vdots & \ddots & \vdots \\
0 & \cdots & 0
\end{array}\right)
$$

where $\mathfrak{U}_{j+1} \mathfrak{L}_{j+1}, \mathfrak{D}_{j} \mathfrak{L}_{j+1}$ are $\ell_{j+1} \times \ell_{j}$ and $\ell_{j} \times \ell_{j}$ matrices, respectively. The blocks $\mathfrak{U}_{j} \mathfrak{A}_{j}$ are $\ell_{j} \times \ell_{j}$ matrices, such that

$$
\mathfrak{U}_{j} \mathfrak{A}_{j}=\left(\begin{array}{cccccc}
-\alpha & 1 & 0 & \cdots & 0 & 0 \\
0 & -\alpha & 1 & \ddots & \vdots & \vdots \\
\vdots & \ddots & \ddots & \ddots & 0 & 0 \\
0 & \cdots & 0 & -\alpha & 1 & 0 \\
a_{1}^{(j)} & a_{2}^{(j)} & \cdots & a_{\ell_{j}-2}^{(j)} & a_{\ell_{j}-1}^{(j)}-2 \alpha & 1 \\
-\mathfrak{u}_{j} & 0 & \cdots & 0 & 0 & 0
\end{array}\right), \mathfrak{D}_{j} \mathfrak{A}_{j+1}=\mathfrak{D}_{j}
$$

Hence, we can rewrite the matrix $\mathfrak{J}^{(p)}=\mathfrak{U} \mathfrak{L}+\alpha I$ as follows

$$
\mathfrak{J}^{(p)}=\mathfrak{U} \mathfrak{L}+\alpha I=\left(\begin{array}{ccccc}
\mathfrak{C}_{\mathfrak{p}_{0}}^{0} & \mathfrak{D}_{0,0} & & & \\
\mathfrak{B}_{1,0} & \mathfrak{C}_{\mathfrak{p}_{0}}^{1} & \mathfrak{D}_{0,1} & & \\
& \mathfrak{B}_{1,1} & \mathfrak{C}_{\mathfrak{p}_{1}}^{0} & \mathfrak{D}_{1,0} & \\
& & \mathfrak{B}_{2,0} & \mathfrak{C}_{\mathfrak{p}_{1}}^{1} & \ddots \\
& & & \ddots & \ddots
\end{array}\right)
$$


where the blocks $\mathfrak{C}_{\mathfrak{p}_{j}}^{0}$ are $\left(\ell_{j}-1\right) \times\left(\ell_{j}-1\right)$ matrices, such that

$$
\mathfrak{C}_{\mathfrak{p}_{j}}^{0}=\left(\begin{array}{ccccc}
0 & 1 & 0 & \cdots & 0 \\
0 & 0 & 1 & \ddots & \vdots \\
\vdots & \ddots & \ddots & \ddots & 0 \\
0 & \cdots & 0 & 0 & 1 \\
a_{1}^{(j)} & a_{2}^{(j)} & \cdots & a_{\ell_{j}-2}^{(j)} & a_{\ell_{j}-1}^{(j)}-\alpha
\end{array}\right)
$$

and the blocks $\mathfrak{D}_{j, 0}, \mathfrak{B}_{j+1,0}$ and $\mathfrak{B}_{j+1,1}$ are $\left(\ell_{j}-1\right) \times 1,1 \times\left(\ell_{j}-1\right)$ and $\left(\ell_{j+1}-1\right) \times 1$ matrices, respectively

$$
\begin{gathered}
\mathfrak{D}_{j, 0}=\left(\begin{array}{c}
0 \\
\vdots \\
0 \\
1
\end{array}\right), \mathfrak{B}_{j+1,0}=\left(\begin{array}{llll}
-\mathfrak{u}_{j} & 0 & \cdots & 0
\end{array}\right), \mathfrak{B}_{j+1,1}=\left(\begin{array}{c}
0 \\
\vdots \\
0 \\
\mathfrak{l}_{j+1}
\end{array}\right), \\
\mathfrak{C}_{\mathfrak{p}_{j}^{1}}^{1}=(\alpha), \mathfrak{D}_{j, 1}=\left(\begin{array}{llll}
1 & 0 & \cdots & 0
\end{array}\right)-\operatorname{are} 1 \times\left(\ell_{j+1}-1\right), j \in \mathbb{Z}_{+} .
\end{gathered}
$$

Therefore, $\mathfrak{J}^{(p)}=\mathfrak{U} \mathfrak{L}+\alpha I$ is the monic generalized Jacobi matrix.

(ii) Assume $\ell_{k-1} \geq 2, \ell_{k}=1$ and $\ell_{k+1} \geq 2, k \in \mathbb{N}$. Then the matrix $\mathfrak{J}^{(p)}=\mathfrak{U} \mathfrak{L}+\alpha I$ has the following representation

$$
\mathfrak{J}^{(p)}=\left(\begin{array}{cccccccc}
\mathfrak{C}_{\mathfrak{p}_{0}}^{0} & \mathfrak{D}_{0,0} & & & & & & \\
\mathfrak{B}_{1,0} & \mathfrak{C}_{\mathfrak{p}_{0}}^{1} & \mathfrak{D}_{0,1} & & & & & \\
& \ddots & \ddots & \ddots & & & & \\
& & \mathfrak{B}_{k, 0} & \mathfrak{C}_{\mathfrak{p}_{k-1}^{1}}^{1} & \mathfrak{D}_{k-1,1} & & & \\
& & & \mathfrak{B}_{k+1,1} & \mathfrak{C}_{\mathfrak{p}_{k}}^{0} & \mathfrak{D}_{k, 0} & & \\
& & & & \mathfrak{B}_{k+2,1} & \mathfrak{C}_{\mathfrak{p}_{k+1}}^{0} & \mathfrak{D}_{k+1,0} & \\
& & & & & \ddots & \ddots & \ddots
\end{array}\right),
$$

where

$$
\left(\begin{array}{cc}
\mathfrak{C}_{\mathfrak{p}_{k-1}}^{1} & \mathfrak{D}_{k-1,1} \\
\mathfrak{B}_{k+1,1} & \mathfrak{C}_{\mathfrak{p}_{k}}^{0}
\end{array}\right)=\left(\begin{array}{cc}
\mathfrak{l}_{k}+\alpha & 1 \\
-\mathfrak{u}_{k} \mathfrak{l}_{k} & -\mathfrak{u}_{k}+\alpha
\end{array}\right)
$$

(iii) Suppose $\ell_{k-1} \geq 2, \ell_{k}=\ldots=\ell_{k+h}=1$ and $\ell_{k+h+1} \geq 2, h, k \in \mathbb{N}$. 
Then we have

$$
\begin{aligned}
& \left(\begin{array}{cccc}
\mathfrak{C}_{\mathfrak{p}_{k-1}^{1}}^{1} & \mathfrak{D}_{k-1,1} & & \\
\mathfrak{B}_{k+1,1} & \mathfrak{C}_{\mathfrak{p}_{k}}^{0} & \ddots & \\
& & \ddots & \mathfrak{D}_{k+h-1,1} \\
& & \mathfrak{B}_{k+h+1,1} & \mathfrak{C}_{\mathfrak{p}_{k+h}}^{0}
\end{array}\right) \\
& =\left(\begin{array}{ccccc}
\mathfrak{l}_{k}+\alpha & 1 & & & \\
-\mathfrak{u}_{k} \mathfrak{l}_{k} & \mathfrak{l}_{k+1}-\mathfrak{u}_{k}+\alpha & 1 & & \\
& \ddots & \ddots & \ddots & \\
& & -\mathfrak{u}_{k+h-1} \mathfrak{l}_{k+h-1} & \mathfrak{l}_{k+h}-\mathfrak{u}_{k+h-1}+\alpha & 1 \\
& & & -\mathfrak{u}_{k+h} \mathfrak{l}_{k+h} & -\mathfrak{u}_{k+h}+\alpha
\end{array}\right) .
\end{aligned}
$$

(iv) Setting $\ell_{0}=\ldots=\ell_{k}=1$ and $\ell_{k+1} \geq 2, k \in \mathbb{Z}_{+}$. We obtain

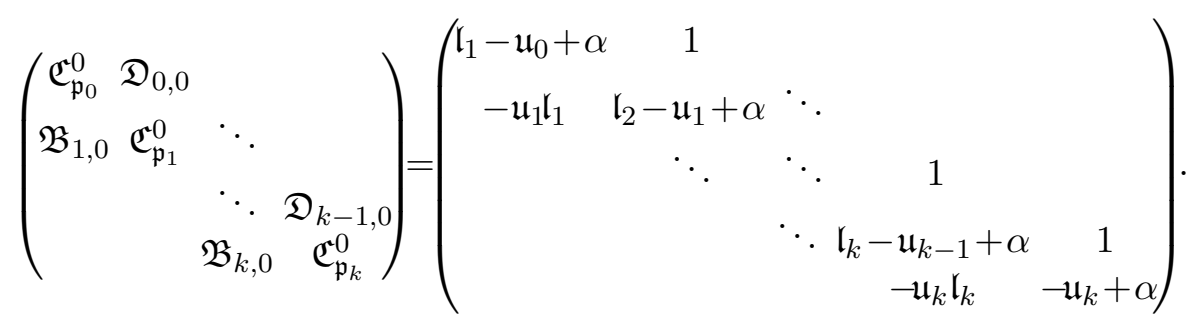

So, from $(i)-(i v)$, we get that $\mathfrak{J}^{(d)}=L U+\alpha I$ is the monic generalized Jacobi matrices. This completes the proof.

Remark 3.1. If $\mathfrak{J}$ is the monic Jacobi matrix, then the $\mathfrak{L} \mathfrak{U}$-factorization of the form (3.1)-(3.3) is coincides with the $L U$-factorization in [2].

Remark 3.2. If $\mathfrak{J}$ is the monic Jacobi matrix of the special form and $\ell_{j}=2$ for each $j \in \mathbb{Z}_{+}$, then the $\mathfrak{L} \mathfrak{U}$-factorization of the form (3.1)-(3.3) is coincides with the $\mathfrak{L} \mathfrak{U}$-factorization in [6].

Remark 3.3. If $\mathfrak{J}$ is the monic generalized Jacobi matrix and $\alpha=0$, then the $\mathfrak{L} \mathfrak{U}$-factorization of the form (3.1)-(3.3) is coincides with the $\mathfrak{L} \mathfrak{U}$-factorization in [15].

Theorem 3.3. Let $\mathfrak{J}$ be the GJM matrix satisfying (3.28) and let $\mathfrak{J}-$ $\alpha I=\mathfrak{L} \mathfrak{U}$ be its $\mathfrak{L} \mathfrak{U}-$ factorization of the form (3.1)-(3.3). Let $\mathfrak{J}^{(p)}=$ $\mathfrak{U} \mathfrak{L}+\alpha I$ be its shifted Darboux transformation without parameter and let

$$
\mathfrak{J}^{(p)} \mathbf{P}^{(p)}(\lambda)=\lambda \mathbf{P}^{(p)}(\lambda),
$$

where $\mathbf{P}^{(p)}(\lambda)=\left(\begin{array}{lllll}P_{0}^{(p)}(\lambda), & P_{1}^{(p)}(\lambda), & \ldots, & P_{\mathfrak{n}_{k}}^{(p)}(\lambda), & \ldots\end{array}\right)^{T}$ 
Then

$$
\begin{aligned}
& P_{\mathfrak{n}_{j}-1}^{(p)}(\lambda)=\frac{1}{\lambda-\alpha}\left(P_{\mathfrak{n}_{j}}(\lambda)-\frac{P_{\mathfrak{n}_{j}}(\alpha)}{P_{\mathfrak{n}_{j-1}}(\alpha)} P_{\mathfrak{n}_{j-1}}(\lambda)\right) \quad j \in \mathbb{N}, \\
& P_{\mathfrak{n}_{j+k}}^{(p)}(\lambda)=\lambda^{k} P_{\mathfrak{n}_{j}}(\lambda), \quad 0 \leq k \leq \ell_{j}-2 \quad \text { and } \quad j \in \mathbb{Z}_{+} .
\end{aligned}
$$

Proof. Let us introduce the following polynomials

$$
\mathbf{P}^{(p)}(\lambda)=\frac{1}{\lambda-\alpha} \mathfrak{U} \mathbf{P}(\lambda)=\frac{1}{\lambda-\alpha}\left(\begin{array}{c}
(\lambda-\alpha) P_{0}(\lambda) \\
\vdots \\
\left(\lambda^{\ell_{0}-1}-\alpha \lambda^{\ell_{0}-2}\right) P_{0}(\lambda) \\
P_{\mathfrak{n}_{1}}(\lambda)-\frac{P_{\mathfrak{n}_{1}}(\alpha)}{P_{\mathfrak{n}_{0}}(\alpha)} P_{\mathfrak{n}_{0}}(\lambda) \\
(\lambda-\alpha) P_{\mathfrak{n}_{1}}(\lambda) \\
\vdots \\
\left(\lambda^{\ell_{1}-1}-\alpha \lambda^{\ell_{1}-2}\right) P_{\mathfrak{n}_{1}}(\lambda) \\
P_{\mathfrak{n}_{2}}(\lambda)-\frac{P_{\mathfrak{n}_{2}}(\alpha)}{P_{\mathfrak{n}_{1}}(\alpha)} P_{\mathfrak{n}_{1}}(\lambda) \\
\vdots \\
\end{array}\right) .
$$

Therefore

$$
\mathfrak{J}^{(p)} \mathbf{P}^{(p)}(\lambda)=\lambda \mathbf{P}^{(p)}(\lambda),
$$

because

$$
\begin{aligned}
\mathfrak{J}^{(p)} \mathbf{P}^{(p)}(\lambda) & =(\mathfrak{U L}+\alpha I) \mathfrak{U} \frac{1}{\lambda-\alpha} \mathbf{P}(\lambda)=\frac{1}{\lambda-\alpha} \mathfrak{H} \mathfrak{J} \mathbf{P}(\lambda) \\
& =\lambda\left(\frac{1}{\lambda-\alpha} \mathfrak{U} \mathbf{P}(\lambda)\right)=\lambda \mathbf{P}^{(p)}(\lambda) .
\end{aligned}
$$

So, the polynomials $P_{h}^{(p)}(\lambda)$ can be represented by the formula (3.40). This completes the proof.

Remark 3.4. If $\ell_{j}=1$ for all $j \in \mathbb{Z}_{+}$, then

$$
P_{\mathfrak{n}_{j}}^{(p)}(\lambda)=\frac{1}{\lambda-\alpha}\left(P_{\mathfrak{n}_{j+1}}(\lambda)-\frac{P_{\mathfrak{n}_{j+1}}(\alpha)}{P_{\mathfrak{n}_{j}}(\alpha)} P_{\mathfrak{n}_{j}}(\lambda)\right)
$$

is a Christoffel formula (see [24]).

Remark 3.5. If at least one $\ell_{j} \geq 2$, then the formula (3.40) is a special case of Christoffel formula (see [24]). 
Theorem 3.4. Let $\mathfrak{J}$ be the GJM satisfying (3.28) and let $\mathfrak{J}-\alpha I=\mathfrak{L} \mathfrak{U}$ be its $\mathfrak{L} \mathfrak{U}$-factorization of the form (3.1)-(3.3). Let $\mathfrak{J}^{(p)}=\mathfrak{U} \mathfrak{L}+\alpha I$ be its shifted Darboux transformation without parameter and let

$$
\left(\mathfrak{J}^{(p)}-\lambda\right) \mathbf{Q}^{(p)}(\lambda)=\Theta_{\ell_{0}-1},
$$

where

$$
\begin{aligned}
& \mathbf{Q}^{(p)}(\lambda)=\left(Q_{0}^{(p)}(\lambda), \ldots, \quad Q_{\mathfrak{n}_{k}}^{(p)}(\lambda), \ldots\right)^{T} \text { and } \\
& \Theta^{(p)}=\left\{\begin{array}{cc}
\Theta_{\ell_{0}-1}=(\underbrace{0, \ldots, 0, \widetilde{b}_{0}}_{\ell_{0}-1}, 0, \ldots), & \text { if } \ell_{0}>1 ; \\
\left(\widetilde{b}_{0}, 0,0, \ldots\right), & \text { if } \ell_{0}=1 .
\end{array}\right.
\end{aligned}
$$

Then

$$
\begin{aligned}
& Q_{\mathfrak{n}_{j}-1}^{(p)}(\lambda)=Q_{\mathfrak{n}_{j}}(\lambda)-\frac{P_{\mathfrak{n}_{j}}(a)}{P_{\mathfrak{n}_{j-1}}(a)} Q_{\mathfrak{n}_{j-1}}(\lambda) \quad j \in \mathbb{N}, \\
& Q_{\mathfrak{n}_{j}+k}^{(p)}(\lambda)=\left(\lambda^{k+1}-\alpha \lambda^{k}\right) Q_{\mathfrak{n}_{j}}^{(p)}(\lambda), \quad 0 \leq k \leq \ell_{j}-2 \text { and } j \in \mathbb{Z}_{+} .
\end{aligned}
$$

Proof. Let us define the following polynomials

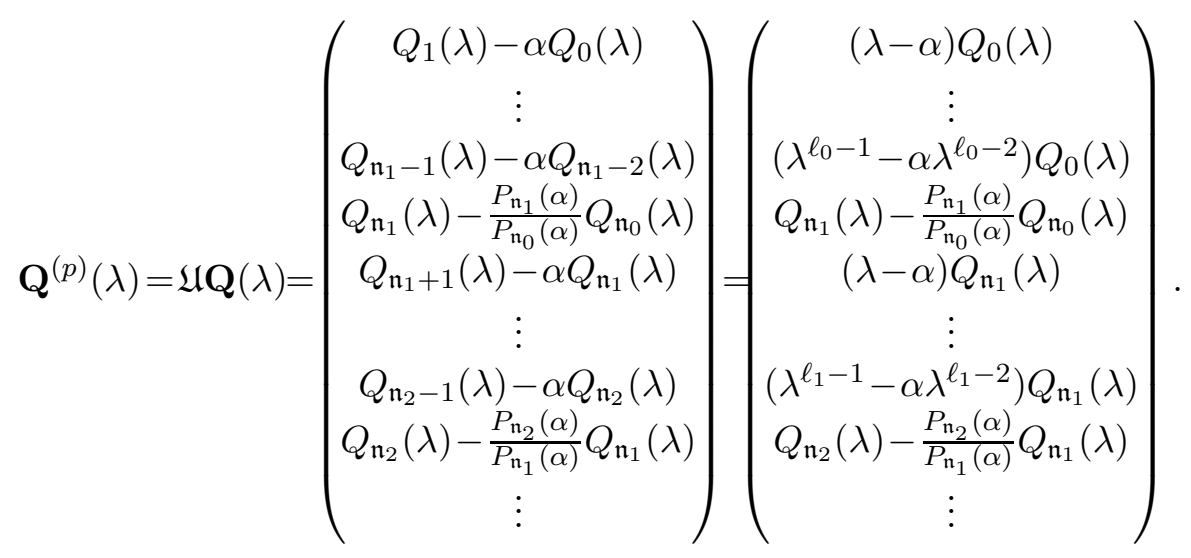

Hence

$$
\begin{aligned}
\left(\mathfrak{J}^{(p)}-\lambda I\right) \mathbf{Q}^{(p)}(\lambda) & =(\mathfrak{U} \mathfrak{L}+(\alpha-\lambda) I) \mathfrak{U} \mathbf{Q}(\lambda) \\
& =\mathfrak{U}(\mathfrak{L} \mathfrak{U}+(\alpha-\lambda) I) \mathbf{Q}(\lambda) \\
& =\mathfrak{U}(\mathfrak{J}+(\alpha-\lambda) I) \mathbf{Q}(\lambda)=\mathfrak{U} \Theta_{\ell_{0}}=\Theta^{(p)} .
\end{aligned}
$$

So, the formula (3.44) is roved. This completes the proof. 
Note the following fact, which is used below: if $\mathfrak{n}_{1}=1$, then

$$
P_{\mathfrak{n}_{1}}(a)=a-\frac{\mathfrak{s}_{1}}{\mathfrak{s}_{0}}=\frac{a \mathfrak{s}_{0}-\mathfrak{s}_{1}}{\mathfrak{s}_{0}} .
$$

On the other hand, by Lemma $3.2, P_{\mathfrak{n}_{1}}(a)=\mathfrak{u}_{0}$, i.e.

$$
\mathfrak{u}_{0}=\frac{a \mathfrak{s}_{0}-\mathfrak{s}_{1}}{\mathfrak{s}_{0}} .
$$

Theorem 3.5. Let $\mathfrak{J}$ be the monic generalized Jacobi matrix associated with the functional $\mathfrak{S}$, such that (3.28) holds and let $\mathfrak{J}-\alpha I=\mathfrak{L U}$ be its $\mathfrak{L} \mathfrak{U}$ - factorization of the form (3.1)-(3.3). Then the matrix $\mathfrak{J}^{(p)}=\mathfrak{U L}+\alpha I$ is associated with the functional

$$
\mathfrak{S}^{(p)}(\lambda)=(\lambda-\alpha) \mathfrak{S}(\lambda) .
$$

Proof. (i) Assume $\mathfrak{n}_{1}>1$. The following equalities hold

$$
\mathfrak{L}_{[0, j-1]}^{T} e_{0}=e_{0} \quad \text { and } \quad \mathfrak{U}_{[0, j-1]} G_{[0, j-1]} e_{0}=b_{0} e_{\mathfrak{n}_{1}-2}, j \in \mathbb{N},
$$

where the shortened matrices $\mathfrak{L}_{[0, j-1]}, \mathfrak{U}_{[0, j-1]}$ and $G_{[0, j-1]}$ are defined analogously to (3.1) and (2.10). Calculating $\mathfrak{s}_{k}$, we get for $j$ large enough

$$
\begin{aligned}
& \mathfrak{S}\left(\lambda^{k}\right)=\mathfrak{s}_{k}=\left[\left(\mathfrak{J}_{[0, j-1]}^{k}\right)^{T} e_{0}, e_{0}\right]=\left(G_{[0, j-1]}\left(\mathfrak{J}_{[0, j-1]}^{k}\right)^{T} e_{0}, e_{0}\right) \\
& =(e_{0}, \underbrace{\left(\mathfrak{L}_{[0, j-1]} \mathfrak{U}_{[0, j-1]}+\alpha\right) \ldots\left(\mathfrak{L}_{[0, j-1]} \mathfrak{U}_{[0, j-1]}+\alpha\right)}_{k \text { times }} G_{[0, j-1]} e_{0}) \\
& =(\mathfrak{L}_{[0, j-1}^{T} e_{0}, \underbrace{\left.\mathfrak{U}_{[0, j-1)} \mathfrak{L}_{[0, j-1]}+\alpha\right) \ldots\left(\mathfrak{U}_{[0, j-1]} \mathfrak{L}_{[0, j-1]}+\alpha\right)}_{k-1 \text { times }} \mathfrak{U}_{[0, j-1]} G_{[0, j-1]} e_{0}) \\
& +(e_{0}, \underbrace{\left(\mathfrak{L}_{[0, j-1]} \mathfrak{U}_{[0, j-1]}+\alpha\right) \ldots\left(\mathfrak{L}_{[0, j-1]} \mathfrak{U}_{[0, j-1]}+\alpha\right)}_{k-1 \text { times }} \alpha G_{[0, j-1]} e_{0}) .
\end{aligned}
$$

Let $\widetilde{G}_{[0, j+n-1]}$ be associated with the matrix $\mathfrak{J}_{[0, j+n-1]}^{(p)}$, where $n$ is the numbers of $\ell_{h}$, such that $\ell_{h} \geq 2,0 \leq h \leq j-1$. It is defined by (2.10). Then $\widetilde{G}_{[0, j+n-1]} e_{0}=b_{0} e_{\mathfrak{n}_{1}-2}$. Using (3.49), we obtain

$$
\begin{aligned}
& =\left(\left(\left(\mathfrak{J}_{[0, j+n-1]}^{(p)}\right)^{T}\right)^{k-1} e_{0}, \widetilde{G}_{[0, j+n-1]} e_{0}\right) \\
& +\alpha\left(\left(\left(\mathfrak{J}_{[0, j-1]}\right)^{T}\right)^{k-1} e_{0}, G_{[0, j-1]} e_{0}\right)=\left[\left(\left(\mathfrak{J}_{[0, j+n-1]}^{(p)}\right)^{T}\right)^{k-1} e_{0}, e_{0}\right] \\
& +\alpha\left[\left(\left(\mathfrak{J}_{[0, j-1]}\right)^{T}\right)^{k-1} e_{0}, e_{0}\right]=\mathfrak{s}_{k-1}^{(p)}+\alpha \mathfrak{s}_{k-1}=\mathfrak{S}^{(p)}\left(\lambda^{k-1}\right)+\alpha \mathfrak{S}\left(\lambda^{k-1}\right) .
\end{aligned}
$$


Therefore

$$
\mathfrak{S}^{(p)}(\lambda)=(\lambda-\alpha) \mathfrak{S}(\lambda) .
$$

(ii) Suppose $\mathfrak{n}_{1}=1$. Then

$$
\mathfrak{L}_{[0, j-1]}^{T} e_{0}=e_{0} \quad \text { and } \quad \mathfrak{U}_{[0, j-1]} G_{[0, j-1]} e_{0}=-\mathfrak{u}_{0} b_{0} e_{0}, j \in \mathbb{N},
$$

Let $\widetilde{G}_{[0, j+n-1]}$ be associated with the matrix $\mathfrak{J}_{[0, j+n-1]}^{(p)}$, where $n$ is the numbers of $\ell_{h}$, such that $\ell_{h} \geq 2,0<h \leq j-1$. The matrix $\widetilde{G}_{[0, j+n-1]}$ is defined by (2.10). Then $\widetilde{G}_{[0, j+n-1]} e_{0}=\frac{\mathfrak{s}_{1}-\alpha \mathfrak{s}_{0}}{\mathfrak{s}_{0}} b_{0} e_{0}$. Calculating

$$
\begin{aligned}
\mathfrak{S}\left(\lambda^{k}\right) & =\frac{\mathfrak{s}_{1}-\alpha \mathfrak{s}_{0}}{\mathfrak{s}_{0}}\left(\left(\left(\mathfrak{J}_{[0, j+n-1]}^{(p)}\right)^{T}\right)^{k-1} e_{0}, \widetilde{G}_{[0, j+n-1]} e_{0}\right) \\
& +\alpha\left(\left(\left(\mathfrak{J}_{[0, j-1]}\right)^{T}\right)^{k-1} e_{0}, G_{[0, j-1]} e_{0}\right)= \\
& =\left[\left(\left(\mathfrak{J}_{[0, j+n-1]}^{(p)}\right)^{T}\right)^{k-1} e_{0}, e_{0}\right]+\alpha\left[\left(\left(\mathfrak{J}_{[0, j-1]}\right)^{T}\right)^{k-1} e_{0}, e_{0}\right] \\
& =\mathfrak{s}_{k-1}^{(p)}+\alpha \mathfrak{s}_{k-1}=\mathfrak{S}^{(p)}\left(\lambda^{k-1}\right)+\alpha \mathfrak{S}\left(\lambda^{k-1}\right) .
\end{aligned}
$$

Hence

$$
\mathfrak{S}^{(p)}(\lambda)=(\lambda-\alpha) \mathfrak{S}(\lambda) .
$$

Due to (3.50) and (3.52), the formula (3.48) is proved. This completes the proof.

Corollary 3.2. Let the moment sequences $\left\{\mathfrak{s}_{j}\right\}_{j=0}^{\infty}$ and $\left\{\mathfrak{s}_{j}^{(p)}\right\}_{j=0}^{\infty}$ be associated with the matrices $\mathfrak{J}$ and $\mathfrak{J}^{(p)}$, respectively. Then

$$
\mathfrak{s}_{j}^{(p)}=\mathfrak{s}_{j+1}-\alpha \mathfrak{s}_{j}
$$

Thus, we obtain that the monic generalized Jacobi matrix $\mathfrak{J}^{(p)}=$ $\mathfrak{U} \mathfrak{L}+\alpha I$ is associated with the moment sequence $\mathfrak{s}^{(p)}=\left\{\mathfrak{s}_{j}^{(p)}\right\}_{j=0}^{\infty}$. Consequently, we can define the set of normal indices of the sequence $\mathfrak{s}^{(p)}$ by

$$
\mathcal{N}\left(\mathfrak{s}^{(p)}\right)=\left\{\mathfrak{n}_{j}^{(p)}: \mathbf{d}_{\mathfrak{n}_{j}^{(p)}}^{(p)} \neq 0\right\}, \mathbf{d}_{\mathfrak{n}_{j}^{(p)}}^{(p)}=\operatorname{det}\left(\begin{array}{ccc}
\mathfrak{s}_{0}^{(p)} & \cdots & \mathfrak{s}_{\mathfrak{n}_{j}^{(p)}-1}^{(p)} \\
\ldots & \cdots & \cdots \\
\mathfrak{s}_{\mathfrak{n}_{j}^{(p)}-1}^{(p)} & \cdots & \mathfrak{s}_{2 \mathfrak{n}_{j}^{(p)}-2}^{(p)}
\end{array}\right)
$$


Proposition 3.1. Let $\mathcal{N}(\mathfrak{s})$ be a set of normal indices associated with the monic generalized Jacobi matrix $\mathfrak{J}$ and let $\mathfrak{J}-\alpha I=\mathfrak{L} \mathfrak{U}$ be its $\mathfrak{L} \mathfrak{U}$ - factorization of the form (3.1)-(3.3). Then the set of normal indices associated with the monic generalized Jacobi matrix $\mathfrak{J}^{(p)}=\mathfrak{L U}+\alpha I$ is

$$
\mathcal{N}\left(\mathfrak{s}^{(p)}\right)=\mathcal{N}(\mathfrak{s}) \cup\left\{\mathfrak{n}_{j}-1: j \in \mathbb{N} \ell_{j-1} \geq 2\right\} .
$$

Proof. Let the monic GJM $\mathfrak{J}$ admits the $\mathfrak{L} \mathfrak{U}$ - factorization of the form (3.1)-(3.3), then, by Theorem 3.1, $P_{\mathfrak{n}_{\mathfrak{j}}}(\alpha) \neq 0$. Calculating

$$
P_{\mathfrak{n}_{j}}(\alpha)=\frac{1}{\mathbf{d}_{\mathfrak{n}_{j}}}\left|\begin{array}{cccc}
\mathfrak{s}_{0} & \mathfrak{s}_{1} & \cdots & \mathfrak{s}_{\mathfrak{n}_{j}} \\
\cdots & \cdots & \cdots & \cdots \\
\mathfrak{s}_{\mathfrak{n}_{j}-1} & \mathfrak{s}_{\mathfrak{n}_{j}} & \cdots & \mathfrak{s}_{2 \mathfrak{n}_{j}-1} \\
1 & \alpha & \cdots & \alpha^{\mathfrak{n}_{j}}
\end{array}\right|
$$

we multiply the $k$-th column by $-\alpha$ and add it to the $k+1$-th column, $k=\overline{0, \mathfrak{n}_{j}-1}$, then we obtain

1) if $\ell_{0}>1$, then

$$
\begin{aligned}
& P_{\mathfrak{n}_{j}}(\alpha)=\frac{1}{\mathbf{d}_{\mathfrak{n}_{j}}}\left|\begin{array}{cccc}
\mathfrak{s}_{0} & \mathfrak{s}_{1}-\alpha \mathfrak{s}_{0} & \ldots & \mathfrak{s}_{\mathfrak{n}_{j}}-\alpha \mathfrak{s}_{\mathfrak{n}_{j}-1} \\
\ldots & \ldots & \ldots & \ldots \\
\mathfrak{s}_{\mathfrak{n}_{j}-1} & \mathfrak{s}_{\mathfrak{n}_{j}}-\alpha \mathfrak{s}_{\mathfrak{n}_{j}-1} & \ldots & \mathfrak{s}_{2 \mathfrak{n}_{j}-1}-\alpha \mathfrak{s}_{2 \mathfrak{n}_{j}-2} \\
1 & 0 & \cdots & 0
\end{array}\right| \\
& =\frac{(-1)^{\mathfrak{n}_{j}} \mathbf{d}_{\mathfrak{n}_{j}^{(p)}}^{(p)}}{\mathbf{d}_{\mathfrak{n}_{j}}} \neq 0
\end{aligned}
$$

2) if $\ell_{0}=1$, then

$$
P_{\mathfrak{n}_{j}}(\alpha)=\left(\frac{\mathfrak{s}_{0}}{\mathfrak{s}_{1}-\alpha \mathfrak{s}_{0}}\right)^{-\mathfrak{n}_{j}} \frac{(-1)^{\mathfrak{n}_{j}}}{\mathbf{d}_{\mathfrak{n}_{j}}} \mathbf{d}_{\mathfrak{n}_{j}^{(p)}}^{(p)} \neq 0
$$

By Proposition 3.15 in [15], we get (3.56). This completes the proof.

Corollary 3.3. Let $\mathcal{N}(\mathfrak{s})$ and $\mathcal{N}\left(\mathfrak{s}^{(p)}\right)$ be sets of normal indices associated with the matrices $\mathfrak{J}=\mathfrak{L} \mathfrak{U}+\alpha I$ and $\mathfrak{J}^{(p)}=\mathfrak{U} \mathfrak{L}+\alpha I$, respectively. If $\ell_{j-1}=\mathfrak{n}_{j}-\mathfrak{n}_{j-1} \geq 2$ and $\mathfrak{n}_{0}=0$, for all $j \in \mathbb{N}$. Then

$$
\mathcal{N}\left(\mathfrak{s}^{(p)}\right)=\left\{\mathfrak{n}_{1}-1, \mathfrak{n}_{1}, \mathfrak{n}_{2}-1, \mathfrak{n}_{2}, \ldots\right\} .
$$

Corollary 3.4. Let $\mathcal{N}(\mathfrak{s})$ and $\mathcal{N}\left(\mathfrak{s}^{(p)}\right)$ be sets of normal indices associated with the matrices $\mathfrak{J}=\mathfrak{L} \mathfrak{U}+\alpha I$ and $\mathfrak{J}^{(p)}=\mathfrak{U} \mathfrak{L}+\alpha I$, respectively. If $\ell_{j-1}=\mathfrak{n}_{j}-\mathfrak{n}_{j-1}=1$ and $\mathfrak{n}_{0}=0$, for all $j \in \mathbb{N}$. Then

$$
\mathcal{N}(\mathfrak{s})=\mathcal{N}\left(\mathfrak{s}^{(p)}\right) .
$$


Proposition 3.2. Let $\mathfrak{J}$ be the monic generalized Jacobi matrix associated with the linear functional $\mathfrak{S}$, such that (3.28) holds and let $\mathfrak{J}-\alpha I=\mathfrak{L} \mathfrak{U}$ be its $\mathfrak{L} \mathfrak{U}-$ factorization of the form (3.1)-(3.3). Let $m(\lambda)$ and $m^{(p)}(\lambda)$ be the $m$-functions of the matrices $\mathfrak{J}$ and $\mathfrak{J}^{(p)}$, respectively. Then

$$
m_{[0, j+n-1]}^{(p)}(\lambda)=(\lambda-\alpha) m_{[0, j-1]}(\lambda),
$$

where $n$ is the number of $\ell_{i}$ of the matrix $\mathfrak{J}$, such that $\ell_{i} \geq 2$ and $i=$ $\overline{0, j-1}$.

Proof. Let $n$ be the number of $\ell_{i} \geq 2$, where $i=\overline{0, j-1}$ and let $\widetilde{G}_{[0, j+n-1]}$ be associated with $\mathfrak{J}_{[0, j+n-1]}^{(p)}$. It is defined by $(2.10)$.

(i) Let $\mathfrak{n}_{1}>1$. Then $\mathfrak{s}_{0}=0, \widetilde{G}_{[0, j+n-1]} e_{0}=b_{0} e_{\mathfrak{n}_{1}-2}$ and the equalities (3.49) hold. Calculating

$$
\begin{aligned}
& (\lambda-\alpha) m_{[0, j-1]}(\lambda)=(\lambda-\alpha)\left[\left(\mathfrak{J}_{[0, j-1]}^{T}-\lambda\right)^{-1} e_{0}, e_{0}\right] \\
& =-\left[\left(\mathfrak{L}_{[0, j-1]}^{T} \mathfrak{U}_{[0, j-1]}^{T}+\alpha-\lambda\right)\left(\left(\mathfrak{L}_{[0, j-1]}^{T} \mathfrak{U}_{[0, j-1]}^{T}+\alpha-\lambda\right)^{-1} e_{0}, e_{0}\right]\right. \\
& +\left[\left(\mathfrak{L}_{[0, j-1]}^{T} \mathfrak{U}_{[0, j-1]}^{T}\left(\mathfrak{J}_{[0, j-1]}^{T}-\lambda\right)^{-1} e_{0}, e_{0}\right]\right. \\
& =-\mathfrak{s}_{0}+\left(e_{0},\left(\mathfrak{L}_{[0, j-1]} \mathfrak{U}_{[0, j-1]}+\alpha-\bar{\lambda}\right)^{-1} \mathfrak{L}_{[0, j-1]} \mathfrak{U}_{[0, j-1]} G_{[0, j-1]} e_{0}\right) \\
& =\left(e_{0}, \mathfrak{L}_{[0, j-1]}\left(\mathfrak{U}_{[0, j-1]} \mathfrak{L}_{[0, j-1]}+\alpha-\bar{\lambda}\right)^{-1} e_{\ell_{0}-2}\right) \\
& =\left(\left(\left(\mathfrak{J}_{[0, j+n-1]}^{(p)}\right)^{T}-\lambda\right)^{-1} e_{0}, \widetilde{G}_{[0, j+n-1]} e_{0}\right)=m_{[0, j+n-1]}^{(p)}(\lambda) .
\end{aligned}
$$

(ii) Let $\mathfrak{n}_{1}=1$. Then $\widetilde{G}_{[0, j+n-1]} e_{0}=\frac{\mathfrak{s}_{1}-\alpha \mathfrak{s}_{0}}{\mathfrak{s}_{0}} b_{0} e_{0}$ and the equalities (3.51) hold. Computing

$$
\begin{aligned}
&(\lambda-\alpha) m_{[0, j-1]}(\lambda)=-\mathfrak{s}_{0}+\left[\left(\mathfrak{J}_{[0, j-1]}^{T}-\lambda\right)^{-1} e_{0}, \mathfrak{L}_{[0, j-1]} \mathfrak{U}_{[0, j-1]} e_{0}\right] \\
&=-\mathfrak{s}_{0}+\left(\left(\mathfrak{J}_{[0, j-1]}^{T}-\lambda\right)^{-1} e_{0}, \mathfrak{L}_{[0, j-1]} \mathfrak{U}_{[0, j-1]} G_{[0, j-1]} e_{0}\right) \\
&=-\mathfrak{s}_{0}+\frac{\mathfrak{s}_{1}-\alpha \mathfrak{s}_{0}}{\mathfrak{s}_{0}}\left(e_{0}, \mathfrak{L}_{[0, j-1]}\left(\mathfrak{U}_{[0, j-1]} \mathfrak{L}_{[0, j-1]}+\alpha-\bar{\lambda}\right)^{-1} e_{0}\right) \\
&=-\mathfrak{s}_{0}+\left[\left(\left(\mathfrak{J}_{[0, j+n-1]}^{(p)}\right)^{T}-\lambda\right)^{-1} e_{0}, e_{0}\right]=-\mathfrak{s}_{0}+m_{[0, j+n-1]}^{(p)}(\lambda) .
\end{aligned}
$$


Thus, we have

$$
m_{[0, j+n-1]}^{(p)}(\lambda)=(\lambda-\alpha) m_{[0, j-1]}(\lambda)+\mathfrak{s}_{0}
$$

So, the formula (3.61) is proved. This completes the proof.

Corollary 3.5. Let $\mathfrak{J}$ be the monic Jacobi matrix and let $\mathfrak{J}^{(p)}$ be the shifted Darboux transformation without parameter of $\mathfrak{J}$. Then

$$
(\lambda-\alpha) Q_{\mathfrak{n}_{j}}(\lambda) P_{\mathfrak{n}_{j}}(\lambda)^{(p)}-Q_{\mathfrak{n}_{j}}^{(p)}(\lambda) P_{\mathfrak{n}_{j}}(\lambda)=\mathfrak{s}_{0} P_{\mathfrak{n}_{j}}^{(p)}(\lambda) .
$$

Proof. Due to the representation (2.14)

$$
m_{[0, j-1]}(\lambda)=-\frac{Q_{\mathfrak{n}_{j}}(\lambda)}{P_{\mathfrak{n}_{j}}(\lambda)} \quad \text { and } \quad m_{[0, j+n-1]}^{(p)}(\lambda)=-\frac{Q_{\mathfrak{n}_{j}}^{(p)}(\lambda)}{P_{\mathfrak{n}_{j}}^{(p)}(\lambda)}
$$

and by Proposition 3.2, we get (3.62). This completes the proof.

\section{Examples}

In this section, we consider the some examples of the shifted Darboux transformations. Recall some facts

Definition 4.1. A function $F \in \mathbf{N}_{-\infty}$ if and only if

1. $F$ is holomorphic on $\mathbb{C}_{+}$,

2. $\operatorname{Im} F(\lambda) \geq 0$ for all $\lambda \in \mathbb{C}_{+}$,

3. $F$ admits the following asymptotic expansion

$$
F(\lambda)=-\frac{\mathfrak{s}_{0}}{\lambda}-\frac{\mathfrak{s}_{1}}{\lambda^{2}} \cdots-\frac{\mathfrak{s}_{2 n}}{\lambda^{2 n+1}}+o\left(\frac{1}{\lambda^{2 n+1}}\right), \quad \lambda \widehat{\rightarrow} \infty,
$$

with $\mathfrak{s}_{j} \in \mathbb{R}$ for all $j \in \mathbb{Z}_{+}$, where $\lambda \widehat{\rightarrow} \infty$ means that $\lambda$ tends to $\infty$ nontangentially, that is inside the sector $\varepsilon<\arg \lambda<\pi-\varepsilon$ for some $\varepsilon>0$.

As was known [14,25], every function $F \in \mathbf{N}_{-\infty}$ admits the $J$-fraction expansion

$$
F(\lambda) \sim-\frac{b_{0}}{\lambda-c_{0}-\frac{b_{1}}{\lambda-c_{1}-\frac{b_{2}}{\ddots}}}=-\frac{b_{0}}{\lambda-c_{0}}-\frac{b_{1}}{\lambda-c_{1}}-\cdots .
$$

Obviously, $F\left(\lambda^{3}\right)$ has the following asymptotic expansion

$$
F\left(\lambda^{3}\right)=-\frac{\widetilde{\mathfrak{s}}_{0}}{\lambda}-\frac{\widetilde{\mathfrak{s}}_{1}}{\lambda^{2}} \cdots-\frac{\widetilde{\mathfrak{s}}_{2 n}}{\lambda^{2 n+1}}-\cdots, \quad \lambda \widehat{\rightarrow} \infty,
$$


where $\widetilde{\mathfrak{s}}_{3 j+2}=\mathfrak{s}_{j}$ and $\widetilde{\mathfrak{s}}_{j}=0$ otherwise. On the other hand, the expansion (4.3) can be rewritten as the $P$-fraction (see [19])

$$
F\left(\lambda^{3}\right) \sim-\frac{b_{0}}{\lambda^{3}-c_{0}}-\frac{b_{1}}{\lambda^{3}-c_{1}}-\frac{b_{2}}{\lambda^{3}-c_{2}}-\cdots .
$$

Hence, $F\left(\lambda^{3}\right)$ is associated with the monic generalized Jacobi matrix

$$
\mathfrak{J}=\left(\begin{array}{cccc}
\mathfrak{C}_{\mathfrak{p}_{0}} & \mathfrak{D}_{0} & & \\
\mathfrak{B}_{1} & \mathfrak{C}_{\mathfrak{p}_{1}} & \mathfrak{D}_{1} & \\
& \mathfrak{B}_{2} & \mathfrak{C}_{\mathfrak{p}_{2}} & \ddots \\
& & \ddots & \ddots
\end{array}\right)
$$

where the entries are defined by

$$
\mathfrak{C}_{\mathfrak{p}_{j}}=\left(\begin{array}{ccc}
0 & 1 & 0 \\
0 & 0 & 1 \\
c_{j} & 0 & 0
\end{array}\right), \mathfrak{D}_{j}=\left(\begin{array}{ccc}
0 & 0 & 0 \\
0 & 0 & 0 \\
1 & 0 & 0
\end{array}\right) \text { and } \mathfrak{B}_{j+1}=\left(\begin{array}{ccc}
0 & 0 & 0 \\
0 & 0 & 0 \\
b_{j+1} & 0 & 0
\end{array}\right) \text {. }
$$

Example 1a. Now we study the shifted Darboux transformation without parameter of the GJM $\mathfrak{J}$ associated with the function $F\left(\lambda^{3}\right)$.

Let $P_{\mathfrak{n}_{j}}(\lambda)$ be the polynomials of the kind associated with the GJM $\mathfrak{J}$. Let us choose some $\alpha \in \mathbb{R}$, such that

$$
P_{\mathfrak{n}_{j}}(\alpha) \neq 0 .
$$

By Theorem 3.1, $\mathfrak{J}$ admits the $\mathfrak{L} \mathfrak{U}$ - factorization (3.1)-(3.3), i.e.

$$
\mathfrak{L}=\left(\begin{array}{ccc}
\mathfrak{A}_{0} & 0 & \\
\mathfrak{L}_{1} & \mathfrak{A}_{1} & \ddots \\
& \ddots & \ddots
\end{array}\right) \quad \text { and } \quad \mathfrak{U}=\left(\begin{array}{ccc}
\mathfrak{U}_{0} & \mathfrak{D}_{0} & \\
0 & \mathfrak{U}_{1} & \ddots \\
& \ddots & \ddots
\end{array}\right) \text {, }
$$

$\mathfrak{D}_{j}$ are defined by (4.6) $\mathfrak{A}_{j}, \mathfrak{L}_{j+1}, \mathfrak{U}_{j}$ take the form (see (3.1)- (3.3))

$$
\mathfrak{A}_{j}=\left(\begin{array}{crr}
1 & 0 & 0 \\
0 & 1 & 0 \\
-\alpha^{2} & -\alpha & 1
\end{array}\right), \mathfrak{L}_{j+1}=\left(\begin{array}{ccc}
0 & 0 & 0 \\
0 & 0 & 0 \\
0 & 0 & -\frac{b_{j+1}}{\mathfrak{u}_{j}}
\end{array}\right), \mathfrak{U}_{j}=\left(\begin{array}{ccc}
-\alpha & 1 & 0 \\
0 & -\alpha & 1 \\
-\mathfrak{u}_{j} & 0 & 0
\end{array}\right),
$$

where $\mathfrak{u}_{0}=-c_{0}-\alpha^{3}$ and $\mathfrak{u}_{i}=-\frac{b_{i}}{\mathfrak{u}_{i-1}}-c_{i}-\alpha^{3}, i \in \mathbb{N}$. Then

$$
\mathfrak{J}^{(p)}=\mathfrak{U} \mathfrak{L}+\alpha I=\left(\begin{array}{cccc}
\mathfrak{C}_{0}^{0} & \mathfrak{D}_{0,0} & & \\
\mathfrak{B}_{1,0} & \mathfrak{C}_{0}^{1} & \mathfrak{D}_{0,1} & \\
& \mathfrak{B}_{1,1} & \mathfrak{C}_{1}^{0} & \ddots \\
& & \ddots & \ddots
\end{array}\right)
$$


is the shifted Darboux transformation without parameter of $\mathfrak{J}$ (see Theorem 3.2). Furthermore, the entries have the following form

$$
\begin{aligned}
& \mathfrak{C}_{j}^{0}=\left(\begin{array}{cc}
0 & 1 \\
-\alpha^{2} & -\alpha
\end{array}\right), \mathfrak{D}_{j, 0}=\left(\begin{array}{l}
0 \\
1
\end{array}\right), \mathfrak{B}_{j+1,1}=\left(\begin{array}{c}
0 \\
-\frac{b_{j+1}}{\mathfrak{u}_{j}}
\end{array}\right), \\
& \mathfrak{B}_{j+1,0}=\left(\begin{array}{ll}
-\mathfrak{u}_{j} & 0
\end{array}\right), \mathfrak{C}_{j}^{1}=(\alpha), \mathfrak{D}_{j, 1}=\left(\begin{array}{ll}
1 & 0
\end{array}\right), \quad j \in \mathbb{Z}_{+} .
\end{aligned}
$$

By Corollary $3.2, \mathfrak{s}^{(p)}=\left\{\mathfrak{s}_{j}^{(p)}\right\}_{j=0}^{\infty}$ is associated with the GJM $\mathfrak{J}^{(p)}=\mathfrak{U L}+\alpha I$, where $\mathfrak{s}_{3 j}^{(p)}=0, \quad \mathfrak{s}_{3 j+1}^{(p)}=\mathfrak{s}_{j}, \quad \mathfrak{s}_{3 j+2}^{(p)}=-\alpha \mathfrak{s}_{j}, j \in \mathbb{Z}_{+}$. Consequently, the function $F^{(p)}(\lambda)=(\lambda-\alpha) F\left(\lambda^{3}\right)$ is associated with the monic generalized Jacobi matrix $\mathfrak{J}^{(p)}=\mathfrak{U} \mathfrak{L}+\alpha I$.

Example 1b. This example is a special case of the Example 1a. We consider the monic Chebyshev-Hermite polynomials $\left\{H_{k}(\lambda)\right\}_{k=0}^{\infty}$ and study the shifted Darboux transformation without parameter of the monic generalized Jacobi matrix $\mathfrak{J}$ associated with $\left\{H_{k}\left(\lambda^{3}\right)\right\}_{k=0}^{\infty}$.

Let $\mathfrak{s}=\left\{\mathfrak{s}_{j}\right\}_{j=0}^{\infty}$ be a moment sequence corresponding to the measure $e^{-t^{2}} d t$ on $\mathbb{R}$, i.e.

$$
\mathfrak{s}_{0}=\sqrt{\pi}, \quad \mathfrak{s}_{2 j}=\frac{\sqrt{\pi}}{2^{j}}(2 j-1) ! ! \quad \text { and } \quad \mathfrak{s}_{2 j-1}=0, \quad j \in \mathbb{N} .
$$

Then the corresponding recurrence relation takes the form

$$
\lambda H_{k}(\lambda)=H_{k+1}(\lambda)+\frac{k}{2} H_{k-1}(\lambda), \quad \text { for all } k \in \mathbb{N},
$$

and the corresponding polynomials of the first kind coincide with the monic Chebyshev-Hermite polynomials

$$
H_{j}(\lambda)=\frac{(-1)^{j}}{2^{j}} e^{\lambda^{2}} \frac{\mathrm{d}^{j}}{\mathrm{~d} \lambda^{j}}\left(e^{-\lambda^{2}}\right) \quad \text { for all } j \in \mathbb{Z}_{+},
$$

where $x \in(-\infty,+\infty)$ and the polynomials $H_{j}(\lambda)$ are orthogonal in $L_{2}(\mathbb{R}, w(\lambda))$ with the weight function $w(\lambda)=e^{-\lambda^{2}}$.

Let us consider the sequence of the polynomials $\left\{H_{j}\left(\lambda^{3}\right)\right\}_{j=0}^{\infty}$ which satisfies the recurrence relation

$$
\lambda^{3} H_{k}\left(\lambda^{3}\right)=H_{k+1}\left(\lambda^{3}\right)+\frac{k}{2} H_{k-1}\left(\lambda^{3}\right), \quad \text { for } k \in \mathbb{N} .
$$

The polynomials $\left\{H_{j}\left(\lambda^{3}\right)\right\}_{j=0}^{\infty}$ are polynomials of the first kind associated with the monic generalized Jacobi matrix $\mathfrak{J}$ defined by (4.5)-(4.6). 
Consequently, the moment sequence $\widetilde{\mathfrak{s}}=\left\{\widetilde{\mathfrak{s}}_{j}\right\}_{j=0}^{\infty}$ associated with the matrix $\mathfrak{J}$ takes the form

$$
\widetilde{\mathfrak{s}}_{3 j}=\widetilde{\mathfrak{s}}_{3 j+1}=0, \quad \widetilde{\mathfrak{s}}_{3 j+2}=\mathfrak{s}_{j}, \quad j \in \mathbb{Z}_{+} .
$$

Let us choose $\alpha \in \mathbb{R}$, such that

$$
H_{j}\left(\alpha^{3}\right) \neq 0 \quad \text { for all } j \in \mathbb{Z}_{+} .
$$

Then $\mathfrak{J}-\alpha I$ admits the $\mathfrak{L} \mathfrak{U}$-factorization (4.9)-(4.10), i.e.

$$
\mathfrak{J}-\alpha I=\mathfrak{L} \mathfrak{U} .
$$

Therefore, the monic generalized Jacobi matrix

$$
\mathfrak{J}^{(p)}=\mathfrak{U} \mathfrak{L}+\alpha I .
$$

is the shifted Darboux transformation without parameter of $\mathfrak{J}$ (see Example 1a).

By Theorem 3.3, the polynomials $\left\{P_{j}^{(p)}(\lambda)\right\}_{j=0}^{\infty}$ of the first kind associated with the matrix $\mathfrak{J}^{(p)}$ are given by

$$
\begin{aligned}
& P_{3 j}^{(p)}(\lambda)=H_{j}\left(\lambda^{3}\right), \quad P_{3 j+1}^{(p)}(\lambda)=\lambda H_{j}\left(\lambda^{3}\right), \\
& P_{3 j+2}^{(p)}(\lambda)=\frac{1}{\lambda-\alpha}\left(H_{j+1}\left(\lambda^{3}\right)-\frac{H_{j+1}(\alpha)}{H_{j}(\alpha)} H_{j}\left(\lambda^{3}\right)\right), \quad j \in \mathbb{Z}_{+} .
\end{aligned}
$$

\section{References}

[1] N. I. Akhiezer, The classical moment problem, Oliver and Boyd, Edinburgh, 1965.

[2] M. I. Bueno, F. Marcellán, Darboux transformation and perturbation of linear functionals // Linear Algebra Appl., 384 (2004), 15-242.

[3] M. Castro, F. A. Grunbaum, The Darboux process and time-and-band limiting for matrix orthogonal polynomials // Linear Algebra Appl. 487 (2015), 328-341.

[4] T. S. Chihara, An Introduction to Orthogonal Polynomials, Gordon and Breach, New York, 1978.

[5] M. Derevyagin, Generalized Jacobi operators in Krein spaces, J // Math. Annal. Appl., 349 (2009), 568-582.

[6] M. Derevyagin, On the relation between Darboux transformations and polynomial mappings // Journal of Approximation Theory, 172 (2013), 4-22.

[7] M. Derevyagin, V.Derkach, Spectral problems for generalized Jacobi matrices // Linear Algebra Appl., 382 (2004), 1-24. 
[8] M. Derevyagin, V.Derkach, Darboux transformations of Jacobi matrices and Páde approximation // Linear Algebra and Its Applications, 435 (2011), No. 12, 30563084 .

[9] V. Derkach, M. Malamud, The extension theory of Hermitian operators and the moment problem // J.of Math.Sci., 73 (1995), No. 2 , 141-242.

[10] V. Derkach, I. Kovalyov, On a class of generalized Stieltjes continued fractions // Methods of Funct. Anal. and Topology, 21 (2015), No. 4, 315-335.

[11] A. Duran, F. A. Grunbaum, A survey on orthogonal matrix polynomials satisfying second order dierential equations // J. Comput. Appl. Math., 178 (2005), 169190.

[12] Ya. L. Geronimus, On the polynomials orthogonal with respect to a given number sequence // Zap. Mat. Otdel. Khar'kov. Univers. i Nil Mat. i Mehan., 17 (1940), $3-18$.

[13] F. Gesztesy, B.Simon, m-functions and inverse spectral analysis for finite and semi-infinite Jacobi matrices // Journal d'Analyse Math., 73 (1997), 267-297.

[14] W. B. Jonec, W. J. Thron, Continued fraction, Addison-Wesley Company, London-Amsterdam, 1980.

[15] I. Kovalyov, Darboux transformation of generalized Jacobi matrices // Methods of Funct. Anal. and Topology, 20 (2014), No. 4, 301-320.

[16] I. Kovalyov, Darboux transformation with parameter of generalized Jacobi matrices // J.Math. Sci., 222 (2017), No. 6, 703-722.

[17] I. Kovalyov, Darboux transformation of the Laguerre operator // Complex Analysis and Operator Theory, 12 (2018), No. 3, 787-809.

[18] P. Lancaster, Theory of Matrices, Academic Press, NY, 1969.

[19] A. Magnus, Certain continued fractions associated with the Padé table // Math. Zeitschr., 78 (1962), 361-374.

[20] M. Malamud, On a formula of the generalized resolvents of a nondensely defined Hermitian operator // Ukr. Mat. Zh., 44 (1992), No. 12, 1658-1688.

[21] F. Peherstorfer, Finite perturbations of orthogonal polynomials // J. Comput. Appl. Math., 44 (1992), 275-302.

[22] V. Spiridonov, A. Zhedanov, Discrete Darboux transformations, the discretetime Toda lattice, and the Askey-Wilson polynomials // Methods Appl. Anal., 2 (1995), No. 4, 369-398.

[23] V. Spiridonov, A. Zhedanov, Self-similarity, spectral transformations and orthogonal and biorthogonal polynomials in self-similar systems, in : V.B. Priezzhev, V.P. Spiridonov (Eds.), Proc. Internat. Workshop JINR, Dubna, 1999, pp. 349361. 
[24] G. Szegö, Orthogonal Polynomials, fourth edition, AMS, 1975.

[25] H. S. Wall, Analytic Theory of Continued Fractions. D. Van Nostrand Company, Inc., New York, N. Y., 1948.

[26] A. Zhedanov, Rational spectral transformations and orthogonal polynomials // J. Comput. Appl. Math., 85 (1997), No. 1, 67-86.

\section{CONTACT INFORMATION}

Ivan M. Kovalyov Dragomanov National

Pedagogical University,

Kiev, Ukraine

E-Mail: i.m.kovalyov@gmail.com 